\title{
An Educational Reconstruction of Special Relativity Theory for Secondary Education
}

\author{
Floor Kamphorst ${ }^{1,2}$ (D) M. J. Vollebregt ${ }^{3} \cdot$ E. R. Savelsbergh ${ }^{1,4}$ D \\ W. R. van Joolingen ${ }^{1}$ (D)
}

Accepted: 8 September 2021 / Published online: 18 October 2021

(c) The Author(s) 2021

\begin{abstract}
Einstein's derivation of special relativity theory (SRT), based on hypothetical reasoning and thought experiments, is regarded as a prime example of physics theory development. In secondary education, the introduction of SRT could provide a great opportunity for students to engage in physics theorizing, but this opportunity is largely being missed in current teaching practice. One reason could be that secondary students lack some knowledge of electromagnetism that was central to Einstein's argument. Therefore, we conducted an educational reconstruction to develop a teaching approach that would not rely on advanced understanding of electromagnetism, yet retain the modes of reasoning that were characteristic of Einstein's approach. In our reconstruction, we identified the light postulate, which is notoriously difficult for students to grasp, as a central concept. We developed a teaching and learning sequence in which students perform relativistic thought experiments and try different interpretations of the light postulate. Through these activities, students experienced how the new concepts meet the requirements for a good theory. Experimental evaluation of the teaching and learning sequence indicates that this can be a fruitful approach to introduce SRT to secondary students.
\end{abstract}

\section{Introduction}

Special relativity theory (SRT) has recently been introduced to secondary physics curricula in several countries. One of these countries is the Netherlands, where SRT became part of the pre-university level physics curriculum in 2014. The theory has an iconic, pop-culture status among the public, which might inspire enthusiasm and curiosity in future learners.

Floor Kamphorst

f.kamphorst@uu.nl

1 Freudenthal Institute for Science and Mathematics Education, Utrecht University, P.O. Box 85170, 3508 AD Utrecht, the Netherlands

2 Gemeentelijk Gymnasium Hilversum, Vaartweg 54, 1217 SV Hilversum, the Netherlands

3 St. Bonifatius College, Burg. Fockema Andrealaan 7-9, 3582 KA Utrecht, the Netherlands

4 Research Centre for Learning and Innovation, Utrecht University of Applied Sciences, P.O. Box 14007, 3508 SB Utrecht, the Netherlands 
More importantly, SRT revolutionized the way physicists look at the world and is a prototypical example of theory development in physics. Therefore, SRT is a promising topic to familiarize students with physics as a process of scientific knowledge development, a key element in the history and philosophy of science. Science education aims for students to gain insight in the process of theory development, in addition to the aim for conceptual understanding (College voor Toetsen en Examens, 2018; National Research Council, 2012; OECD, 2013). However, this opportunity is not seized in the conventional textbook presentations of SRT, and little is known about how to achieve this.

Although SRT provides a prototypical example of physics theory development, its introduction may be challenging for secondary students. First, the historical reasons why SRT was introduced and how the theory was developed draw on prior knowledge that is not generally part of the secondary curriculum. Furthermore, the abstract and counterintuitive concepts and outcomes of SRT are difficult to learn (Gousopoulos et al, 2016; Hewson, 1982; Scherr et al., 2002; Villani \& Pacca, 1987). SRT represents a transformation in physics, giving radically new meaning to existing concepts. Among other things, the theory replaced the classical concepts of an absolute time and space with an observer dependent spacetime. Gaining a type of knowledge that requires the learner to revise their basic assumptions is notoriously difficult and often leads to misconceptions (Vosniadou, 1994). Therefore, in a teaching and learning sequence (TLS), it is important to explicitly connect to students' prior knowledge, and support them in giving new, relativistic interpretations to familiar concepts (for example Amin \& Levrini, 2017; Driver et al., 1994; Kattmann et al., 1996; Posner et al., 1982; van Oers \& Wardekker, 1997; Vosniadou, 1994).

To design such a TLS, we found a productive tool in the Model of Educational Reconstruction (MER). MER provides a design frame to bridge the gap between students' ideas and physics concepts (Duit et al., 2012; Komorek \& Duit, 2004). To this end, the model focusses specifically on the history and philosophy of physics to inform the educational design. Therefore, this design framework fits our learning aim with its dual focus on both conceptual understanding and the development of physics theories. (For a more extensive discussion on MER, see Section 2.1).

In this article, we present an educational reconstruction of SRT and a first proof of principle of the resulting teaching approach as an answer to the following research question:

How can learners in secondary education develop a conceptual understanding of SRT through engaging in a process of physics theory development?

a. How can the key ideas and theory development of SRT be reconstructed into a content structure for instruction?

b. To what extent can a TLS based on the aforementioned content structure be successful to bridging student ideas and physics concepts?

The first sub-question will be answered in Section 2. After a brief presentation of MER, we will present our analyses of SRT from both the theory and the student perspective, resulting in the reconstructed content structure of SRT for secondary education. The second sub-question will be answered in the third and fourth section. The third section describes the teaching and learning sequence based on the proposed content structure and the rationale how the design may contribute to our overall learning aim. The fourth section describes the empirical evaluation of the design, illustrating whether the expected learning is also observed in a practical situation. We will conclude with answering our main research question and discussing some of the implications of this study. 




Fig. 1 The three components of research of the Model of Educational Reconstruction

\section{Educational Reconstruction}

\subsection{Model of Educational Reconstruction}

MER is a specific approach to design-based research. Specific for this approach is that the educational design is informed both by an analysis of the theory and its history, and by an analysis of the learners' perspective in an iterative design process (Duit et al., 2012; Komorek \& Duit, 2004). Figure 1 shows how the analyses of the theory perspective and the learners' perspective mutually influence each other and the design and evaluation of learning environments in an iterative process.

The analysis from the theory perspective aims to clarify the conceptual structure of the domain and to help identify the key insights to be attained from the perspective of the overall learning goal. Analysis of the history and philosophy of the domain can help to identify likely conceptual hurdles, and ways of overcoming them.

The analysis of the learners' perspective aims to identify relevant prior knowledge and learning difficulties for the core elements of the theory. In addition, this analysis also seeks out successful approaches to overcome these difficulties. To this end, we will draw on the available research literature.

The previous analyses result in a breakdown of the theory in its basic elements and learning difficulties with these concepts, and ways to overcome them. The reconstruction rebuilds the theory from a learners' perspective, resulting in a content structure for instruction. This content structure serves as a guide for the conceptual development of the learner towards the relativistic concepts. This reconstruction serves as a starting point for an educational design and its evaluation.

In the following sections, we will first report our analysis from the theoretical perspective, and the learner perspective, in order to attempt an educational reconstruction in Section 2.4, which will be built of insights from both perspectives. 


\subsection{Analysis from the Theoretical Perspective}

Here we present an analysis of the theory from the perspective of the overall learning goal. The Dutch curriculum describes the following learning goal for SRT: "The candidate can explain the phenomena of time dilation and length contraction, using the concepts of light speed, reference frame and simultaneity, in the contexts of thought experiments and applications" (College voor Toetsen en Examens, 2018). We will take this aim as a starting point for our analysis. The emphasis on explaining relativistic phenomena invites a conceptual approach. Therefore, we will in addition aim to contribute to students' scientific literacy by reflecting the process of theory development in our design. To these ends, we will identify the basic principles of SRT and analyze the reasoning that led to these principles and the theory itself. In our analysis, we build upon insights from the philosophy of sciences, in particular the work by Lakatos (1976), which will be outlined below, and the styles of scientific reasoning described by Kind and Osborne (2017).

The process of students constructing a new understanding can bear interesting similarities to the process the scientific community went through in accepting the original idea (for example Gopnik \& Wellman, 2012; Posner et al., 1982; Vosniadou, 1994). Posner et al. (1982), for instance, described how their theory of conceptual change was similar to the process of scientific theory development as described by Lakatos (1976). Lakatos argues that scientific theories can be regarded as part of a research program in which the successive theories constitute a consistently progressive theoretical shift over their predecessors (Lakatos, 1976). New theories are accepted in the scientific community because of this progressive theoretical shift. According to Lakatos, such theories meet five requirements:

1. There is a need for a new way of looking at the world;

2. The new theory is plausible and intelligible;

3. The new theory solves the problems that cause the need to look at the world in a new way;

4. The new theory confirms what is already known; and

5. The new theory leads to a fruitful research program.

Posner argues that, although there are many differences between scientific experts and novices, there can also be fruitful parallels, in that both scientists and students will tend to stick to their old ideas, and they will only change to a new idea if specific conditions have been met. Therefore, educational designers may find helpful clues in the history and philosophy of physics to help students bridge the gap between their preinstructional ideas and physics concepts (Kattmann et al., 1996; Levrini, 2014). This is not to argue that the history of science should be replicated in the classroom, but rather that clues from history and philosophy can be one element to inform the educational reconstruction of the material. In our case, we are searching for an educational reconstruction that will retain the essential characteristics of the reasoning process that led to the development of SRT. As Kind and Osborne (2017) argued, each discipline has its own characteristic style of reasoning. They propose six characteristic styles to deserve a place in secondary education: mathematical deduction, experimental evaluation, hypothetical modelling, categorization and classification, probabilistic reasoning, and historical-based evolutionary reasoning. We propose that the frameworks proposed by Lakatos and by Kind and Osborne can be helpful to capture the essential feature of 
the process that led to the development and acceptance of SRT. Therefore, we will use these frameworks as a lens to analyze the development of SRT as presented by Einstein.

SRT builds on two existing theories: Maxwell's electrodynamics and Galileo relativity. In the paper "Zur Elektrodynamik bewegter Körper" (Einstein, 1905), Einstein addressed a mismatch between the principles of Galilean relativity and the interpretation of Maxwell's electrodynamics. From this mismatch, which Einstein referred to as an asymmetry, he inferred the need for a new way of looking at the world:

It is known that Maxwell's electrodynamics - as usually understood at the present time-when applied to moving bodies, leads to asymmetries which do not appear to be inherent in the phenomena. Take, for example, the reciprocal electrodynamic action of a magnet and a conductor. The observable phenomenon here depends only on the relative motion of the conductor and the magnet, whereas the customary view draws a sharp distinction between the two cases in which either the one or the other of these bodies is in motion. (Einstein, 1905/1952b, p. 37)

The asymmetry Einstein referred to pertains to the phenomenon of induced current in a coil. When a permanent magnet moves relative to a coil of conducting material, a current will be induced in the coil. This process can be described from the reference frame of a stationary coil with a moving magnet, or from the reference frame of a stationary magnet with a moving coil. The induced current is the same from both perspectives. That natural phenomena are independent of the reference frame you describe them from was widely accepted in classical mechanics. Galileo described in his theory of relativity that the mechanical phenomena on a ship are not affected by its state of motion. Moreover, it was widely accepted that the theoretical explanations causing these phenomena are also independent of reference frame, i.e., the laws of mechanics are invariant under transformation to a different (inertial) reference frame. One would expect, as Einstein did, that the mechanics causing the current to run in the coil is also independent of the choice of reference frame. However, the interpretation of Maxwell's theory in Einstein's time was different. If the phenomenon was described from the frame of the stationary coil and the moving magnet, the current was caused by an induced electric field and thus an electric force, and if the phenomenon was described from the frame of the stationary magnet and the moving coil, the current was caused by a magnetic force. Einstein argued that there is no reason in the observed phenomena to accept this theoretical difference:

Examples of this sort, together with the unsuccessful attempts to discover any motion of the earth relatively to the "light medium," suggest that the phenomena of electrodynamics as well as of mechanics possess no properties corresponding to the idea of absolute rest. They suggest rather that, as has already been shown to the first order of small quantities, the same laws of electrodynamics and optics will be valid for all frames of reference for which the equations of mechanics hold good. (Einstein, 1905/1952b, pp. 37-38)

Here, Einstein introduced his epistemic conviction that there is a need to describe the world in a new way (Requirement 1), which can be interpreted as a desire for symmetry or unification: not only should the laws of mechanics be invariant under transformation, Galilean relativity should be expanded to the domain of electromagnetism and optics (Abiko, 2005). The new theory should solve the asymmetries in Maxwell's Electrodynamics Einstein referred to. To this end, Einstein introduced a new way of describing light propagation, the light postulate: 
We will raise this conjecture (the purport of which will hereafter be called the "Principle of Relativity") to the status of a postulate, and also introduce another postulate, which is only apparently irreconcilable with the former, namely, that light is always propagated in empty space with a definite velocity $c$ which is independent of the state of motion of the emitting body. These two postulates suffice for the attainment of a simple and consistent theory of the electrodynamics of moving bodies based on Maxwell's theory for stationary bodies. The introduction of a "luminiferous ether" will prove to be superfluous inasmuch as the view here to be developed will not require an "absolute stationary space" provided with special properties, nor assign a velocity-vector to a point of the empty space in which electrodynamics takes place."

(Einstein, 1905/1952b, pp. 37-38)

From the current perspective on Maxwell's electromagnetism, the light postulate does not seem a far stretch. However, it was revolutionary in Einstein's time. Maxwell's equations follow an electromagnetic wave that propagates with a velocity $c$. Experiments performed by Hertz had demonstrated that light had electromagnetic properties, and therefore, the electromagnetic wave proposed by Maxwell was interpreted to be light. However, the theory did not specify a reference frame for this wave. Before Einstein, light propagation was considered a mechanical process, and light velocity was implicitly or explicitly defined relative to a source or a medium. Newton, for instance, considered light to have a corpuscular nature (Newton, 1730/1952), implying a constant speed relative to the light source. In this model, light is described as a stream of tiny particles. By contrast, Huygens compared light propagation to sound waves (Huygens, 1690/1952). This wave-like model describes light as a mechanical wave propagating with a constant speed relative to a medium or rest frame. The nineteenth century physicists assumed a medium was needed for electromagnetic waves as well: the luminiferous ether.

However, about twenty years before Einstein's work, the attempt by Michelson and Morley to demonstrate the movement of the earth relative to this medium had failed: they could not detect evidence of relative movement (Michelson \& Morley, 1887; Lorentz, $1895 / 1952 a)$. Einstein referred to this experiment as "unsuccessful attempts" to prove the existence of the luminiferous ether. ${ }^{1}$ He proposed to follow Maxwell and to break with the practice of specifying a reference frame relative to which the speed of light is constant. Rather, he proposed the speed of light is constant, and equal to c, in all reference frames. It is therefore not surprising Einstein assumed the light postulate might seem contradictory for his audience. However, Einstein promised a "simple and concise theory" that would solve the mentioned asymmetries in (the interpretation of) Maxwell's electromagnetic theory and expand it to the relativity principle. In our view, Einstein offered with this a plausible and intelligible (Requirement 2) argument to, at least temporarily, accept the light postulate to be true and find out if Einstein delivers on his promise.

Guided by the constraints of the relativity principle, Einstein derived his new theory from the light postulate "[w]ith the help of certain imaginary experiments" (Einstein, $1905 / 1952$ b, p. 40). He showed what the world would look like when we temporarily assume the light postulate to be true. This became especially clear in Einstein's derivation of a new definition of simultaneity: he took a system of two inertial observers in relative motion, assumed the light postulate to be true, and used deductive reasoning to arrive at a

\footnotetext{
1 Note that Michelson and Morley assumed the luminiferous ether was present, and solely aimed to establish the relative movement of the earth to this medium (Gim, 2016).
} 
new consistent definition of this concept. Likewise, other key concepts of the theory, such as observers, events, time and spatial coordinates were carefully defined in such a way as to comply with the postulates. From these concepts, Einstein derived a set of equations to transform coordinates in spacetime from one inertial frame to another. Einstein's interpretation of these transformations combined the separate concepts of space and time into one unified spacetime. In addition, Maxwell's equations are invariant under these transformations. Thus, SRT solves the asymmetry in the interpretation of electromagnetism by replacing Galilean relativity with a new relativity theory. With that, Einstein showed that the new theory solved the problems that caused the need to look at the world in a new way (Requirement 3).

In order to be accepted, the new theory should also satisfy the other criteria proposed by Lakatos. As it turns out, the theory agrees with what is already known (Requirement 4). The transformations that Einstein presented were previously derived by Lorentz to explain the findings of the Michelson-Morley interferometer experiment (Lorentz, 1904/1952b), and SRT converged to Newtonian mechanics in the limits of low speeds. Furthermore, SRT also offered leads to new lines of investigation (Requirement 5). New phenomena predicted by SRT invited experimental verification. This was true, for example, for the light postulate: the Dutch astronomer de Sitter concluded that light from binary star systems always reached the earth at the same speed, independent of the speed of the light sources (de Sitter, 1913). These results corroborated SRT. Besides, SRT turned out to be just the beginning, describing only the limited domain of inertial frames. In order to complete the theory for situations where acceleration and gravity do play a role, further theoretical efforts were needed, leading to the General Relativity Theory (Einstein, 1916/1952a). At its introduction, SRT was part of a lively scientific debate. The ultimate implications of the theory remained hard to accept for many prominent physicists, such as Lorentz (Klomp, 1997). Despite this, SRT was accepted in the physics community over previous ways of looking at the world (classical physics) because it meets the requirements for a good theory.

In terms of scientific reasoning styles, Einstein's introduction of SRT drew heavily on hypothetical modelling. The process of hypothetical modelling can be described in four stages. First, a model is proposed and temporarily assumed to be correct. For SRT, this is the light postulate. Second, the consequences of the model are derived. Einstein derived relativistic phenomena such as the relativity of simultaneity, time dilation, and length contraction. In addition to the light postulate, he drew on the concepts of observer and inertial reference frame and deductive reasoning in thought experiments. Third, there was a reflection on this result. For SRT specifically, the epistemic value of the Relativity Postulate guided this process. More generally, for the reasoning style of hypothetical modelling, the value of a model as a heuristic tool, the explanatory coherence of the model and the limits to representational accuracy also contribute to accepting the knowledge claims produced. Finally, this reflection resulted in the acceptance or rejection of the model. SRT explained observed phenomena and provided solutions for known problems, which contributed to the acceptance of the theory and the light postulate, even before its experimental verification. This process illustrates that hypothetical modelling (and other styles of scientific reasoning) draws not only on theoretical concepts, but is also informed by procedures to come to a knowledge claim and epistemic values to guide the decision whether to accept the produced knowledge claims or not (Kind \& Osborne, 2017).

The theory development that we want reflected in our design can be summarized by the reason for Einstein to introduce SRT, a seeming conflict between two theories and the desire of unification, and hypothetical modelling with the light postulate. The latter also reflects one of the central principles of SRT. These findings lead us to reformulate our 
learning aim for SRT to productive reasoning with the light postulate, which means students can use the light postulate to derive and explain relativistic concepts.

\subsection{Analysis of the Learners' Perspective}

Here we analyze the theory development and central principles of SRT from a student perspective. First, we will focus on how SRT and the postulates are introduced. Subsequently, we will show what problems students may encounter with the relativistic phenomena derived from these basic concepts. Finally, we will present some solutions for these learning difficulties. This analysis will provide us with leads for our educational reconstruction and design.

Introducing SRT Einstein introduced the need for SRT by addressing an asymmetry with the interpretation of Maxwell's electromagnetism and subsequently expanding Galilean relativity to hold for electromagnetism as well. This historical logic cannot be copied directly into the secondary classroom, for two reasons. First, students, at least in the Netherlands, will not have sufficient knowledge of electrodynamics (see: College voor Toetsen en Examens, 2018). Second, most students do not hold strongly to the relativity principle as a guiding principle for theory development (Bandyopadhyay, 2009; Panse et al., 1994; Pietrocola \& Zylbersztajn, 1999). As we argued in Section 2.2, it is this epistemic feature of the relativity principle that is essential to Einstein's argument. Therefore, we need to find alternative phenomena that can give rise to a similar line of reasoning.

Light Propagation Central to relativity and the introduction of relativistic concepts is productive reasoning with the light postulate. The light postulate is notoriously difficult for students of all levels of education. It is reported that students can recite the light postulate after standard instruction (Dimitriadi \& Halkia, 2012; Guisasola et al., 2009; Yildiz, 2012). However, it is also reported they cannot apply it (Gousopoulos et al., 2016), a prerequisite for productive reasoning. Instead, students use Galilean velocity addition, and they interpret $c$ as the maximum speed that can be attained (Gousopoulos et al., 2016; Villani and Arruda, 1998). These studies show that students know that light has a constant speed, but they do not operationalize it in the formal way of SRT. Instead, students reason with a spontaneous or pre-instructional model of light propagation. In a study by Kamphorst et al. (2019) about students' pre-instructional ideas, secondary students were asked to draw constant light propagation, thinking from the perspective of an observer in various relativistic situations. Rather than drawing and reasoning with a constant speed of light relative to this observer, participants reasoned with a constant speed of light relative to the light source, or relative to a form of absolute space (Kamphorst et al., 2019). Similar ideas have been found after instruction in higher education (Villani and Pacca, 1987). Therefore, it seems difficult to change these pre-instructional ideas.

Relative Motion and Intrinsic Phenomena Relativistic phenomena such as the relativity of simultaneity, time dilation, and length contraction only become apparent when interpreting the same events in two reference frames that are in relative motion. At first glance, relative motions do not seem problematic for students: Bandyopadhyay (2009) and Kamphorst et. al. (2019) found that students compare velocities of objects and compare reference frames or describe light propagation relative to different frames, using spontaneous Galileo transformation. However, Saltiel and Malgrange (1980) found that students also regard 
motion as a property of the object itself. Several studies on students' ideas of reference frames, movement, and classical relativity found that students often compare real motion with a dynamical cause, to apparent motion (Panse et al., 1994; Ramadas et al., 1996; Saltiel \& Malgrange, 1980) and that they often treat this apparent motion as an optical illusion (Panse et al., 1994; Saltiel \& Malgrange, 1980) caused by viewing from another reference frame (Panse et al., 1994). Students also tend to regard other phenomena like length and duration as an intrinsic property of a process or object (Dimitriadi \& Halkia, 2012; Gousopoulos et al., 2016; Hewson, 1982; Levrini, 2008; Saltiel \& Malgrange, 1980; Scherr et al., 2001, 2002; Villani \& Pacca, 1987). Furthermore, Scherr et. al. (2001) found that students do not recognize that relativistic phenomena are a consequence of two frames in relative motion. Students tend to treat the relativity of simultaneity as a phenomenon that is independent of relative motion, or they think simultaneity is absolute, omitting the relativistic aspect all together. Students also tend to think that relativistic phenomena such as time dilation and length contraction are apparent and disappear when corrected for signal travel time (Scherr et al., 2001).

Working with Reference Frames To overcome the idea that concepts such as velocity, length, and duration are an intrinsic property of an object or process, students need to apply the concept of reference frame. Scherr et. al. (2001) showed that graduate and advanced undergraduate students after an undergraduate SRT course do not spontaneously apply the concept of reference frame to determine the time of an event. This may be explained by the problems students experience with the concept of reference frame itself. Students often associate reference frames with concrete objects and regard them as fixed to a physical object (Panse et al., 1994). It appears to be difficult for students to determine what makes a reference frame. Students tend to think that observers at the same position (also those in relative motion) share a reference frame and thus agree on times and order of events (Scherr et al., 2001). At the same time, students tend to think that two distant observers (also those that are not in relative motion) are always in different frames, and therefore do not agree on the time and order of events. This may be because students tend to think that reference frames are limited to the sensory experiences of an observer (Panse et al., 1994; Scherr et al., 2001). Things that cannot be seen by an observer, because they are blocked by another object or are far away, are not part of the reference frame of that observer. To address part of the difficulties with reference frame, Dimitriadi and Halkia (2012) used the phrasing "point of view" of a specific observer.

Comparing Reference Frames Special relativity requires that students make clear distinctions between reference frames. The outcome of their reasoning, like the time interval between events or the order of them, depends on the reference frame the events are described from. It appears that students also tend to take this aspect of relativity in overdrive. Scherr et. al. (2002) reported that students tend to think that different reference frames represent different objective realities. This results in students thinking that an event that happens in one reference frame does not need to happen in another and justify this idea by referring to quantum mechanics. In addition, students tend to think that observers cannot exchange information with other observers (Scherr et al., 2002). Also, they have trouble with the notion of an intelligent observer who can correct for signal travel time. Students associate the time of an observer registering an event with the time the event itself occurred, thus ignoring signal travel time. This results in students thinking that events are simultaneous when observed at the same time, again not taking signal travel time into 
account. When students compare reference frames and do acknowledge that observers can exchange information, they still run into difficulties with time dilation and length contraction. Students tend to have an asymmetrical interpretation of these phenomena (Aslanides \& Savage, 2013): The clock of observer B is running slow for observer A because of time dilation. SRT states that this should be the same the other way around since there is one preferred reference frame. However, students think that for observer B the clock of observer A will run faster.

Overcoming the Abstract Nature of SRT In short, students interpret relativistic phenomena as apparent, have difficulty relating them to a reference frame, and do not correct for signal travel time. A complicating factor in addressing these difficulties is that special relativity phenomena are not directly observable to students. First, because SRT predictions differ from their classical counterpart only at very high velocities, and second because special relativity does only apply to situations without acceleration or gravity. Therefore, relativistic phenomena are abstract in nature and difficult to imagine to students. Several authors have proposed that thought experiments (TEs) can help students to learn new, abstract concepts, and to overcome conceptual barriers (Helm et al., 1985; Velentzas \& Halkia, 2013). TEs are supposed to encourage students to explore the consequences of their ideas in an idealized context, and to make their reasoning explicit (Matthews, 1994). This approach could be well-suited for this topic because TEs played a key role in the introduction and communication of SRT (Einstein, 1961, 1979).

Similarities TE and HM Performing a TE can be regarded as a specific form of hypothetical modelling (HM). The first stage of a TE is to describe a central question, an initial situation, and the rules to be applied. This is similar to defining a model in HM. In the second stage of the TE, the consequences of the basic principles are derived to answer the central question. This is analogous to using the model to make predictions in HM. Finally, the overall conclusions of the thought experiment are interpreted, analogously to reflecting on the predictions and deciding to accept or reject the initial model (Kind \& Osborne, 2017; Reiner \& Burko, 2003). Therefore, thought experiments seem a feasible way for students to engage in hypothetical modelling in the domain of SRT. We intend to use the TEs in this way.

Supporting the Process of Performing a TE To perform relativistic TEs, students have to keep track of many processes and a lot of information in their minds. Students have to reason with an absolute speed of light in the context of two moving reference frames and obtain the outcome of the TE. An external representation can support students in this process. The event diagram (ED) has been used successfully to this end (Kamphorst et al., 2021).

In conclusion, the historical line of reasoning, starting from an apparent contradiction in Maxwell's theory, is not a suitable approach to introduce SRT to students. However, we expect that it is worthwhile to maintain the logic leading to the theory of SRT in our educational reconstruction. Moreover, the central reasoning portrayed in SRT, hypothetical modelling by performing (supported) thought experiments, can help students to gain insight in abstract concepts and productive reasoning with the light postulate. TEs can also help students to reconceptualize velocity as a property relative to a reference frame rather than an intrinsic property of the object itself; to compare reference frames while reasoning about events. 


\subsection{Reconstruction for Secondary Education}

In this section, we will present the reconstruction of the key ideas and theory development of SRT into a content structure for instruction. This content structure will be aimed at students learning to reason productively with the light postulate, which means students can use the light postulate to derive and explain relativistic concepts. We designed this reconstruction with a student in mind who does not hold the same strict epistemic values as scientists, but who does show an appreciation for a coherent world view and a commitment to developing a consistent physics understanding of the world. We expect students to prefer a model that can explain observed phenomena over a model that cannot; to recognize that if two models come to different predictions for the same phenomenon, at least one of them will not be correct, and to acknowledge that a more general model is to be preferred over a model with limited predictive value.

The development of SRT was driven by the inconsistency between two theoretical ideas; this was the reason to introduce the light postulate, which could be demonstrated to solve the inconsistency, and led to a fruitful research program. Therefore, the key idea of our educational reconstruction of SRT is to derive relativistic concepts through productive reasoning with the light postulate. To that end, students need to regard phenomena in relation to reference frame, compare reference frames, and reason in context of high relative speeds. To reconstruct the key ideas and these aspects of the theory development of SRT into a content structure for secondary education, we formulated three principles that served as guidelines for our design. We propose these principles can contribute to productive reasoning with light propagation for secondary students. The design should enable students to experience:

1. a need for a new light propagation model, i.e., the light postulate, and that this need is plausible from their perspective,

2. the light postulate solves the problem introduced by the need for a new propagation model and this new model leads to a fruitful research program, and

3. how new knowledge can be developed through hypothetical modelling activities.

The literature analysis showed that the issue about Maxwell's equations that originally gave rise to the development of SRT is not suitable as a context for secondary education. In addition, the analysis showed that introducing the light postulate by defining it does not result in students reasoning with it. They tend to fall back to a pre-instructional light propagation model. This student model can be described as a constant speed relative to something: the light source or the background (Kamphorst et al., 2019). If we describe the absolute speed of light of the light postulate in the same terms, this is a constant speed relative to all inertial observers.

We propose that students can appreciate the need for a new light propagation model once they attain the following insights:

1. their reasoning about light propagation always is relative to a reference frame;

2. different choices for the reference frame are possible;

3. predictions differ dependent on the choice of reference frame; and

4. their current reasoning leads to wrong/inconsistent predictions. 
Once these insights have been attained, they may start questioning their choice of frame, which makes it plausible to introduce a new propagation model. To subsequently start and continue to reason productively with the light postulate, we propose that the students need to:

5. change the reference frame for light propagation to the reference frame of the observer;

6. experience that this new light propagation model solves the problems they experienced with their pre-instructional models; and

7. explore the consequences of this new propagation model.

We have addressed these two phases of theory development (1-4 and 5-7) in the two parts of our educational design, illustrating how the key ideas and theory development of SRT can be reconstructed into a content structure for instruction.

\subsubsection{Part 1: Introducing the Need for a New Light Propagation Model-Becoming Aware of the Limited Predictive Value of Pre-instructional Light Propagation Models}

We expect that hypothetical modelling with their pre-instructional model in carefully designed tasks can create a need for students to start reasoning with the light postulate and follow the epistemology of the theory development of SRT. The two initial models that most students use correspond to light propagation models physicists held in the past: a wave-like and a particle-like model (see Section 2.2). Since students' initial models tend to be fluid over different contexts (DiSessa, 1996), they are named as these corresponding physics models. This is to ensure both models are addressed, students mean the same when referring to a model, and to stimulate students to reason consistently with the models over different contexts. In addition, a formal model provides common ground and can also be used correct or wrong, whereas a student model is correct by definition. Consistent reasoning will lead to different predictions for each model in contexts with relative movement between light source and observer. By comparing these results to findings from historical experiments, students can experience that both models are inconsistent with empirical findings and therefore have limited predictive value. We expect this inconsistency will provide sufficient reason for students to question the reference frame of both these formal models and, as a consequence, the reference frame of their own light propagation model.

\subsubsection{Part 2: Developing Confidence in the Light Postulate as a Propagation Model for Productive Reasoning}

Students can solve the problem of the limited predictive value by proposing a "new" light propagation model: the light postulate, starting a second cycle of hypothetical modelling. Based on the outcome of the experimental evaluation, students can change the light propagation frame to that of the observer. Because of the introductory activities, this new model will be plausible for students and lead to conclusions that confirm what they already know. Subsequently, students can explore the consequences of the light postulate. We also expect that they can experience the reference frame dependence of relativistic phenomena by deriving these themselves. Moreover, this activity may also help students to accept these 
Table 1 Content structure for instruction and how these conceptual steps relate to tasks in the hypothetical learning trajectory

Content structure for instruction

Hypothetical learning trajectory

Part 1: Introducing the need for a new light propagation model_becoming aware of the limited predictive value of pre-instructional light propagation models

HM with pre-instructional model

1. Becoming aware of the initial light propagation model Task 1: Exploring initial ideas and the role of reference frame in this model;

2. becoming aware of other options for reference frame (through the introduction of formal models) and as a

Task 2: Confronting inconsistencies consequence questioning the reference frame of the initial model;

3. becoming aware that the two formal models, and as a consequence the initial propagation model, do not have predictive value in all context (Requirement 1 of Lakatos);

4. therefore rejecting the reference frame of these models and making it plausible to introduce a new model (Requirement 1 of Lakatos)

Part 2: Developing confidence in the light postulate as a propagation model for productive reasoning

HM with light postulate and exploring its consequences

1. Proposing a new, consistent light propagation model which is therefore plausible: the light postulate

(Requirement 2 of Lakatos);

2. confirming the new model solves the problem of limited predictive value (Requirement 3 and 4 of Lakatos);

3. using the light postulate to make predictions in new contexts (Requirement 5 of Lakatos);

4. deciding to keep using the model;

5. deriving new concepts with the light postulate

Task 4: Proposing the light postulate

(Requirement 5 of Lakatos)

concepts and phenomena despite their counterintuitive nature because they can experience the phenomena are a consequence of the light postulate and relative motion.

We acknowledge that the steps of hypothetical modelling by students may not all happen explicitly. For example, students can communicate their models by using them, and communicate they accept the models by continuing to do so.

The content structure for instruction that is the result of our educational reconstruction of special relativity theory for secondary education is summarized in Table 1.

\section{Hypothetical Learning Trajectory}

In the previous section, we described a content structure for instruction that would provide students with a basis to reason productively with the light postulate. To test whether these steps would represent a feasible learning pathway, we designed a hypothetical learning trajectory - that is, a sequence of tasks and activities, each accompanied by hypotheses about 
what the student would learn, and how this learning would become evident from what the student says and does at that point.

The resulting HLT consists of six subsequent tasks, structured around two thought experiments. Each task consists of a reasoning activity supported with an event diagram, and a reflection on this activity. The current section presents the HLT, which will be subjected to empirical evaluation in the Section 4 (Bakker, 2018; Komorek \& Duit, 2004; Simon, 1995). Sections 3 and 4 together will answer the second sub-research question, whether a teaching and learning sequence based on the content structure for instruction can be successful in bridging the gap between students' ideas and physics concepts.

\subsection{Part 1: Introducing the Need for a New Light Propagation Model-Becoming Aware of the Limited Predictive Value of Pre-instructional Light Propagation Models}

This part introduces the need for students to change their ideas about light propagation. To that end, students engage in a sequence of hypothetical modelling tasks using different light propagation models. Each task offers opportunities to become aware of one's reference frame for light propagation and to start questioning this frame. Students will experience that both the "wave-like" and the "particle-like" model lead to inconsistent results. This finding may introduce the need for a new propagation model or make the need for such a model plausible. We do not expect all students to experience this need at the same moment, but we do expect that the three tasks together will introduce the need to reason in a new way with light propagation for all students.

\subsubsection{Task 1: Exploring Initial Ideas}

Aim Students become aware of their initial light propagation model and that this model is a constant speed relative to something.

Task Students are asked to imagine an observer who simultaneously receives two light flashes, coming from opposite directions, on his measuring device. The flashes are emitted by two lamps that are mounted on a moving cart, passing by the observer on the ground. The assignment for the student is to figure out at what time(s) the lamps emitted the light flashes. The initial settings of the task are shown in an event diagram (ED, Fig. 2), a graphical representation of spacetime. The ED shows the position of objects, observers, and events from a specific reference frame at subsequent moments in time. The bottom picture of Fig. 2 shows the event of two light flashes arriving simultaneously at the observer. The position of the light flash at previous instances can be added by the student. This supports students' stepwise reasoning with light propagation in performing the task (Kamphorst et al., 2021). In all tasks of our design, the speed of light in the ED is set at two squares per time unit. Students are free to choose a point of reference for measuring this speed. After performing the task, the teacher asks the students how they constructed their drawings in the ED and how this portrays a constant speed of light.

Evidence of Learning We expect students to construct a constant velocity either relative to the light source (Fig. 2a) or relative to the background or graph paper (Fig. 2b) (Kamphorst 
a

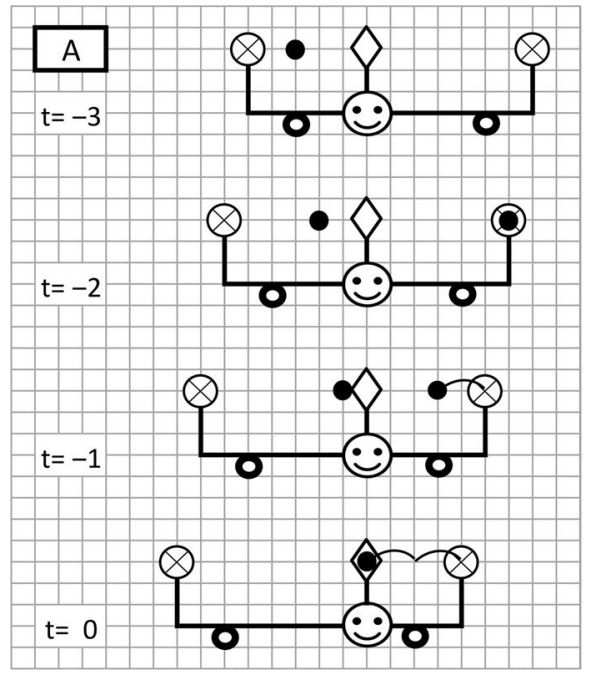

b

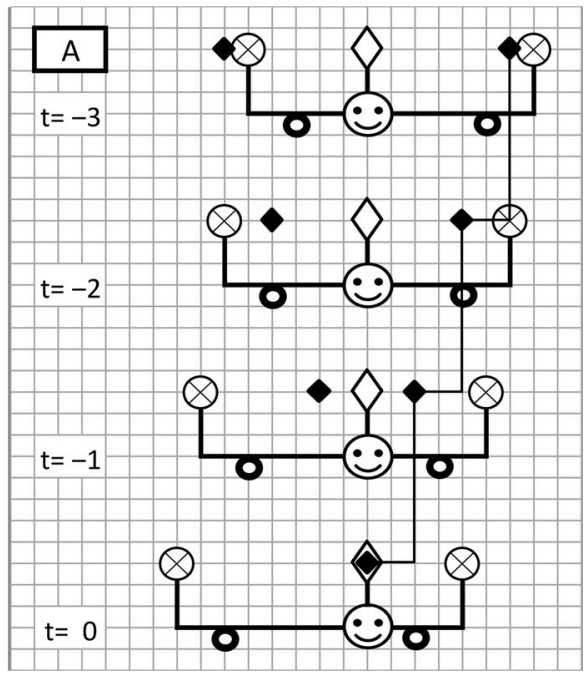

Fig. 2 ED to support Task 1. The figure shows the position of a cart with two lamps (lamp symbol: circle with cross) attached to it at four subsequent time steps. The light flashes emitted by the lamps reach the measuring device of the observer (smiley) simultaneously at $t=0$. The position of the light flash is drawn with dots for two initial light propagation models. a Shows a constant propagation relative to the light source (solid dots); b constant light propagation relative to the graph paper (solid diamonds)

et al., 2019). When the task is performed with a constant speed of light relative to the lamp, the student will predict that the right lamp emitted a light flash at $t=-2$. The second approach predicts that the light flash was emitted at $t=-4$. The task outcome and how this outcome is obtained show students' pre-instructional light propagation model. We expect that the ED supports students in reflecting on their task performance because the ED provides them with a "written" account of their reasoning. We expect that explaining the consistency of their pre-instructional light propagation model makes students aware of this model and that such a model consists of a constant speed relative to something (the lamp or the graph paper). They may show this by mentioning the element in the ED relative to which the speed of light is constant: relative to the lamp, or to the graph paper. By performing and reflecting on this task, students engage in the first two steps of hypothetical modelling: They use a pre-instructional model to make predictions and explicate this model by explaining it to the teacher. If both initial models have been mentioned in the classroom discussion, students might even recognize they can choose between these light propagation frames.

\subsubsection{Task 2: Confronting Inconsistencies}

Aim Students engage in hypothetical modelling with two formal propagation models relative to two reference frames and as a consequence start questioning the frame of their initial propagation model.

Task The teacher introduces two formal propagation models based on the ideas of Newton and Huygens. 
Newton-Particle-Like Model Light propagates with a constant speed relative to the light source. This model is similar to the first student model (Fig. 2a).

Huygens-Wave-Like Model Light propagates with a constant speed relative to a medium or rest frame. The graph paper in the ED can function in the role of medium or rest frame. This model is similar to the second student model (Fig. 2b).

The teacher points out the similarities between the formal models and the student models. Students are asked to repeat the previous thought experiment for each of the formal models. Next, they are asked to use these models in a similar thought experiment where both the observer and the lamps are on the moving cart (Fig. 3b). Again, the two models will lead to different outcomes (Fig. 3). When the task is performed with the particle-like model, students will conclude that the right lamp emitted a light flash at $t=-4$. The wavelike model will lead to the conclusion that the right lamp emitted a light flash at $t=-7$. The teacher reflects on two aspects of the task with the students. First, the teacher asks students to explain for each of the models how it represents a constant speed of light. Second, the teacher asks whether both models could be true at the same time. The teacher confirms that a choice between the models is necessary since an experiment cannot lead to two different outcomes at the same time. ${ }^{2}$

Evidence of Learning We expect that both formal models are plausible for students and that they show this by using both models to perform the thought experiment. Furthermore, we expect students to acknowledge the similarities between their initial model and one of the formal models. Specifically, we expect that students can make the connection between the graph paper in the ED and the medium in the formal wave-like propagation model: the concept of medium in light propagation is part of the secondary curriculum. In addition, we expect students to recognize that both formal models are an example of a constant speed relative to something: either the lamp or the graph paper. They show this by agreeing with the teacher when the models are explained, or by mentioning these reference frames when working with the models. Finally, we expect students to interpret that the two models lead to different task outcomes as that at least one of the models gives a faulty prediction. We expect students will express this by asking which of the two models is true or express a preference for one of the models.

\subsubsection{Task 3: Evaluating Predictions}

Aim Students become aware that the two formal models have limited predictive value and as a consequence reject the reference frames of these propagation models and find it plausible to look for a new propagation model.

Task The students are presented with the outcome of two experiments: the De Sitter experiment (DSE) and the Michelson Morley experiment (MME). Students are asked to identify

\footnotetext{
${ }^{2}$ Some students may assume this is not problematic because of an incorrect interpretation of quantum mechanics. Although the famous double slit experiment in quantum physics confirms the wave nature of electrons or the particle nature when you look through what slit the electron goes, these two different outcomes refer back to two different executions of the experiment. One execution can only confirm one of the models. We do not expect our students to experience this confusion because the topic of quantum mechanics was not covered previous to our intervention.
} 
a

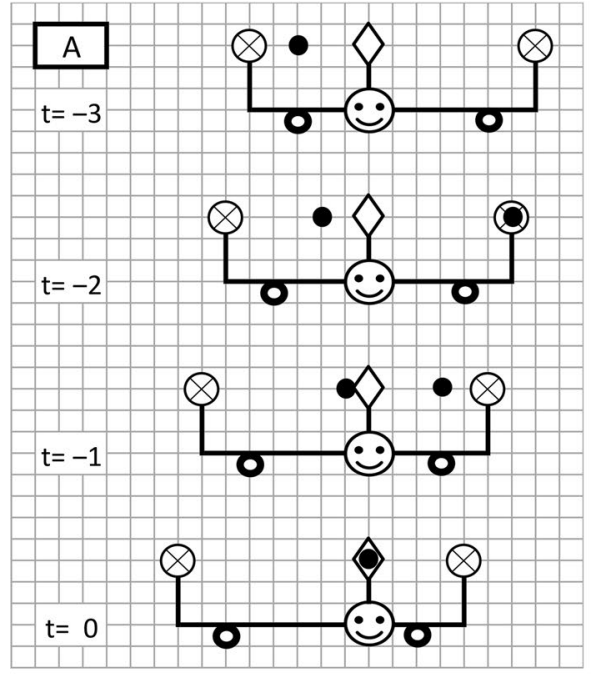

C

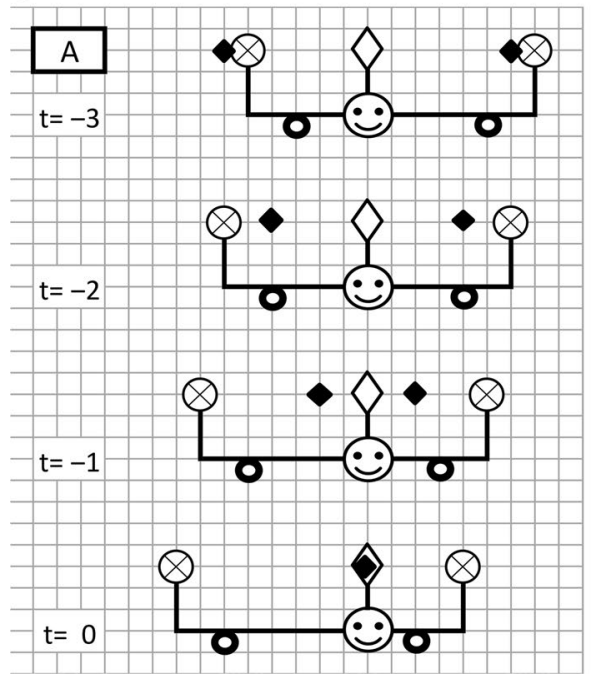

b

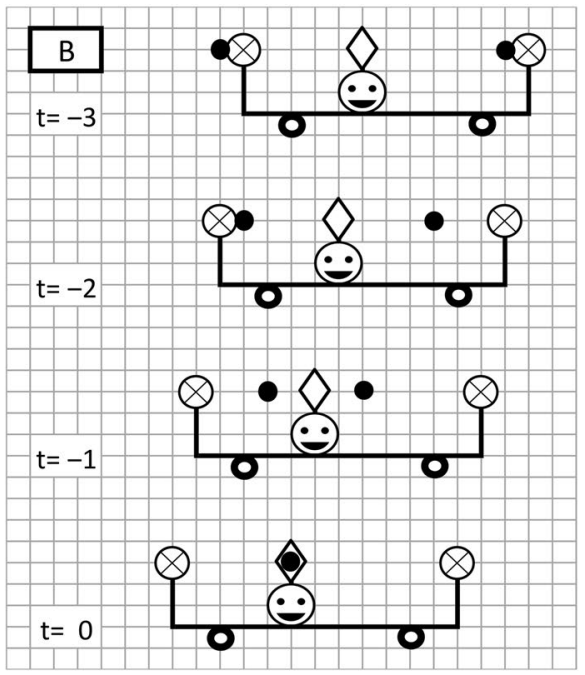

d

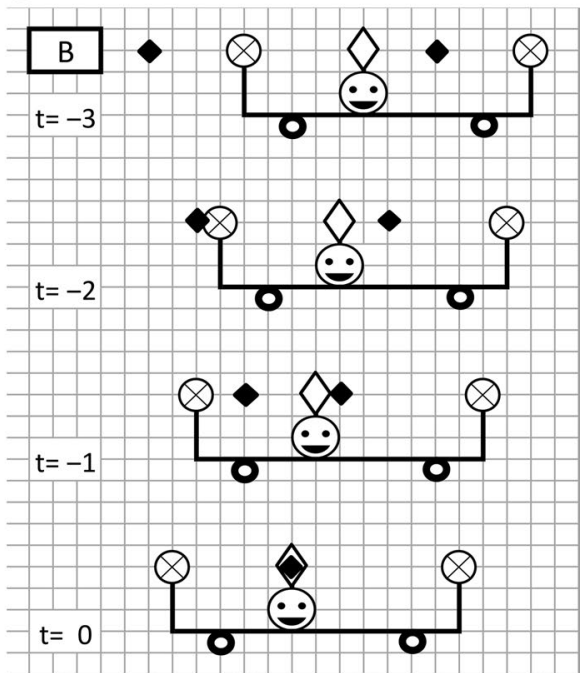

Fig. 3 EDs to support Task 2. a and b Show the task outcome drawn with the particle-like propagation model (dots); $\mathbf{c}$ and $\mathbf{d}$ with the wave-like propagation model (diamonds). Each model gives a different task outcome

which experiment corresponds to what version of the thought experiment in Task 2. To support students in making this connection, they analyze the relative movement between the observer, light source and space or graph paper in both the EDs and the experiments. Once students linked the experiment to the corresponding ED, students are asked to use 
the outcome of the experiments to confirm or reject the predictions made with the formal models.

DSE De Sitter observed light from a binary star system, two stars spinning around a common center of mass. The outcome of this experiment was that all light arrives at the same speed, independent of the direction of movement of the light sources.

The DSE corresponds to ED A as in both situations a stationary observer receives light from two moving light sources. The findings of the DSE correspond to predictions of the wave-like model (Fig. 3c) and not with those of the particle-like model (Fig. 3a).

MME Michelson and Morley measured light from stationary sources while the entire setup moved through space in our planetary orbit. The outcome of this experiment was that light has the same speed, independent of the direction in which the experimental setup moves through space.

The MME corresponds to ED B, as in both situations, the observer is at rest relative to the light sources and this system moves relative to the supposed ether. The findings of the MME correspond to the predictions of the particle-like model (Fig. 3b) and not with those of the wave-like model (Fig. 3d).

Therefore, each formal model is not supported by the outcome of one of the experiments. This overall conclusion provides students with a rationale to reject these propagation models for light. As a consequence, there is a need to introduce a new propagation model. To underline this unexpected overall conclusion of the task, the teacher makes the conclusion formal: Neither formal propagation model gives a coherent description for light propagation that leads to predictions that are confirmed by both experiments in the two situations.

Evidence of Learning We expect students to describe the relative movements in the EDs and we expect this will help them to recognize the similarities between the thought experiments and the DSE and MME. We also expect that students, with some help, can use the interpretation of the experiments to decide which predictions are supported by the experiment and which are falsified. We expect that students, with support from the teacher, will recognize that the task outcome means that neither of the formal models leads to correct predictions in all circumstances. These insights form the third and fourth step of hypothetical modelling: evaluating the predictions and deciding to accept or reject the models. We expect this overall conclusion will introduce the need for a new light propagation model and will make it plausible for students to propose such a model. In addition, we expect the results confirmed by experiments (shown in Fig. 3b and c) to provide students with tools to propose such a model. These expectations are confirmed when students can perform the tasks in Part 2 of the HLT.

\subsection{Part 2: Developing Confidence in the Light Postulate as a Propagation Model for Productive Reasoning}

This part builds on the outcomes of the tasks in the first part. Students use the results of Task 3 to propose a new propagation model with a constant speed relative to the observer: the light postulate. Subsequently, students explore the consequences of the light postulate in new contexts and gain insight in new relativistic concepts, showing they can indeed reason productively with this counterintuitive concept. We expect that students will develop 
an increasing confidence in the light postulate once they see that it leads to a fruitful "research program."

\subsubsection{Task 4: Resolving Tension}

Aim Students propose a new light propagation model and change their light propagation frame to that of the observer. Students recognize that the problems of the limited predictive value of the previous models are solved by this new model.

Task The previous task and the exercise on relative movement provided students with the necessary building blocks to propose the light postulate. Based on the DSE, students should reject constant velocity relative to the light source. Based on the MME, students should reject constant velocity relative to the graph paper. The teacher asks students to propose a propagation rule for light that will reproduce the results of Fig. 3b and c. Subsequently, the teacher asks students to check if their new model indeed reproduces the results of the confirmed predictions in Task 3. To conclude the task, the teacher confirms the new propagation rule: light propagates with a constant speed relative to the observer in all situations.

Evidence of Learning Since the other options have been excluded, we expect students to propose the light postulate in their own wording, for instance: light has a constant speed relative to the observer. This shows students engaged in the first step of hypothetical modelling with the light postulate: proposing a model. We furthermore expect that students will be able to verify that the new model leads to the correct predictions for both the DSE- and MME-results, solving the problem of the limited predictive value of the formal models.

\subsubsection{Task 5: Exploring Counterintuitive Consequences}

Aim Students use the light postulate to perform thought experiments that result in counterintuitive outcomes and discover that the time of an event depends on the reference frame of the observer.

Task Students are asked to find out at what instant the lamps emitted a light flash in two contexts. In the first context, the observer is moving relative to the lamps (ED C, see Fig. 4a). In the second context, a second, stationary observer is introduced (ED D, see Fig. 4b). Students are asked to solve the thought experiment for these observers. Both observers are midway between the lamps and the instant two light flashes arrive simultaneously. Consistent reasoning with the light postulate will lead to the conclusion that the lamps emitted a light flash at $t=-2$ and $t=-6$ for observer $\mathrm{C}$, and that the lamps emitted the light flashes simultaneously at $t=-3$ for observer $\mathrm{D}$. Therefore, the overall task outcome of this TE is that observers in different reference frames will assign different times to the same event. Even more, that events that are simultaneous in the reference frame of one observer, are not simultaneous in the reference frame of another one. Subsequently, the teacher asks students to explain the task outcome. When students explain how the light postulate leads to the task outcomes, they are confronted with the counterintuitive results. At the same time, explaining the relation between the context of the TE, the light postulate and the outcome, can help students to accept this counterintuitive outcome. 
a

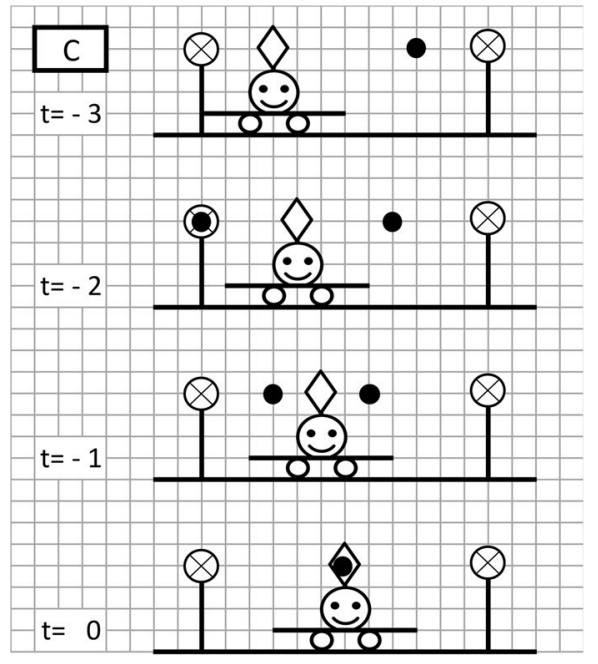

b

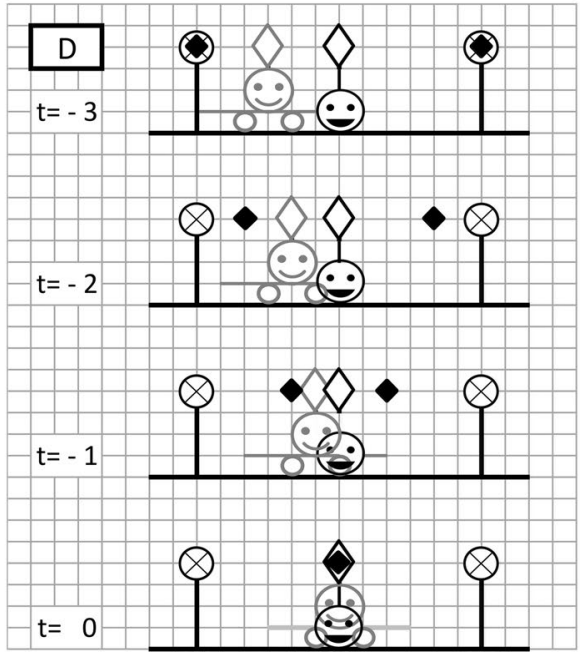

Fig. 4 EDs to support Task 5. Light propagation is drawn with the light postulate for the observer on the cart (a) and the one on the ground (b)

Evidence of Learning We expect students to perform the tasks with the light postulate, although some may fall back to their initial propagation model. If students fall back to their initial model, they will find that the lamps emitted their light flash simultaneously at $t=-3$ for both observers, independent of their initial model. Furthermore, we expect students to express they find these task outcomes counterintuitive. For instance, they would mention that the task outcome is strange. Despite this, we expect students can explain that this outcome is inevitable because the two observers are in relative motion and light has a constant speed relative to both observers. With this explanation, students would show they recognize the relation between the light postulate and the task outcome. Students therefore engage in the second and third step of hypothetical modelling: using the model to make predictions and evaluate these predictions. We expect that the activity of explaining how the task outcomes are a consequence of the light postulate and the relative movement between the observers will help students to accept these counterintuitive outcomes. We expect that in turn, being able to explain the results will also contribute to students to keep using the light postulate in the nest task, showing they accept this new propagation model, which is the fourth step of hypothetical modelling. Students may also express verbally that they accept this new propagation rule.

\subsubsection{Task 6: Exploring the Consequences of the Light Postulate for Time}

Aim Students reason productively with the light postulate by using the light postulate to derive the concept of time dilation and gain a conceptual understanding of this concept.

Task Students perform a version of the light clock thought experiment: 
a

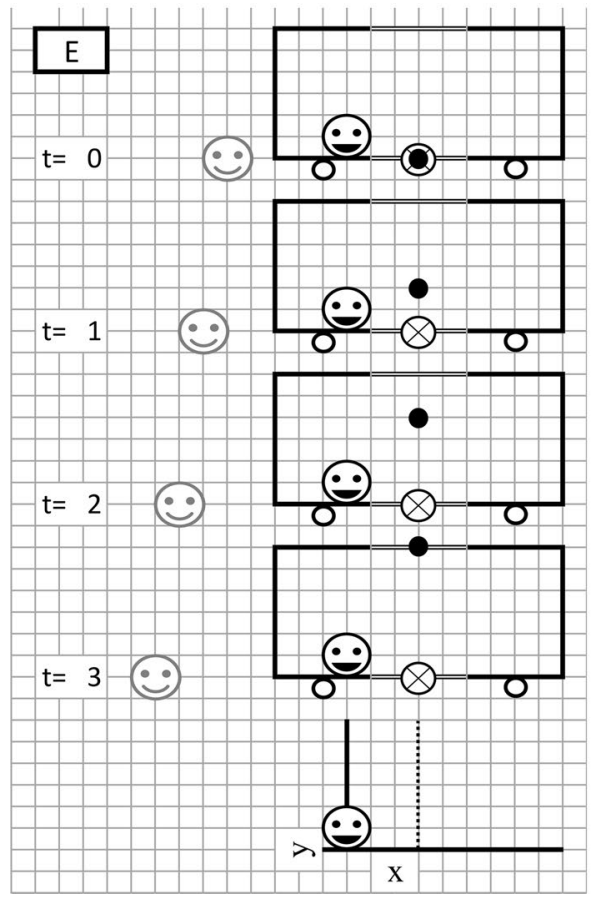

b

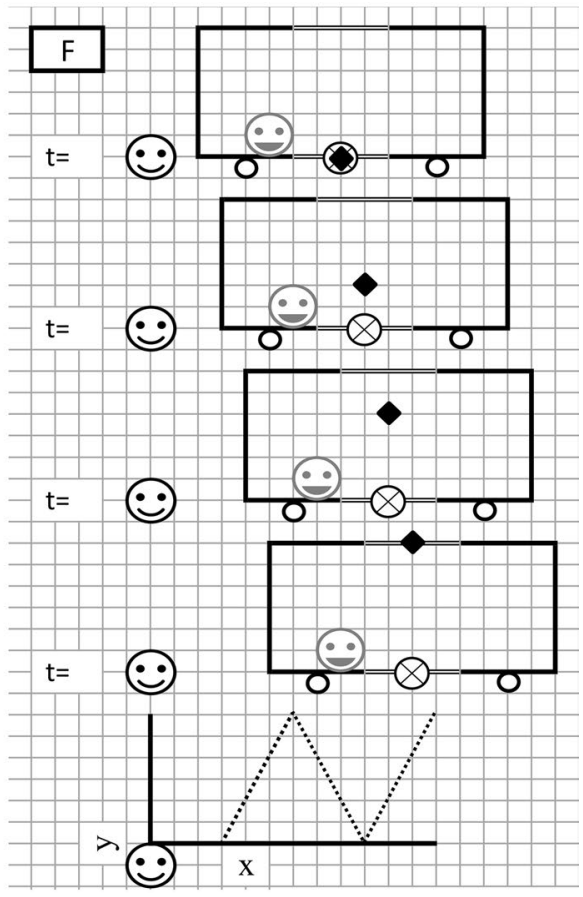

Fig. 5 EDs to support Task 6. The figure shows a light clock drawn from two different reference frames. The light clock consists of two mirrors (horizontal dotted lines) and a lamp in the bottom mirror (symbol of lamp in electrical circuits). a Shows the results when the light postulate is applied for observer A studying mirrors that are stationary in his frame (dots). b Shows the same process for observer B who studies mirrors that are moving relative to his reference frame (diamonds). In that case, the speed of light relative to observer B is bigger than 2 squares per picture. Therefore, the time steps are left blank

Light Clock Thought Experiment A light flash bounces up and down between two mirrors. Two observers study this process. The first observer is stationary relative to the mirrors. The second observer observes the mirrors moving relative to him.

Students are asked to find out how many time steps the light flash needs to bounce up and down between the mirrors according to each observer. The thought experiment is supported with two EDs. Each ED is drawn from the reference frame of one of these observers (Fig. 5). If students reason consistently with the light postulate, they will find that light travels vertically up and down relative to the observer studying mirrors in rest and that light travels a longer distance relative to the observer studying moving mirrors. Both light paths are represented in Fig. 5. The overall outcome of this thought experiment is that light needs more time steps to travel up and down in the light clock for the observer in ED F. To support students in obtaining the overall outcome of the task, the teacher asks them what the difference in path length means. The extent to which time dilation occurs depends on the relative velocity between the two observers. To reflect on this aspect, the teacher asks students how the duration would change in case the velocity between the observers increases or decreases. 
Evidence of Learning We expect students to use the light postulate to interpret the outcome of the thought experiment. By doing so, students again confirm they accept this new model (step four of hypothetical modelling). We expect students to mention that light needs 4 time steps to travel up and down between the mirrors for the observer studying the stationary light clock and we expect students to mention that light needs more than 4 time steps to travel up and down for the observer studying the moving light clock. We expect students to conclude that the duration of the process is longer for the observer studying the moving system. In contrast, students who prefer the classical idea that time intervals are absolute will say that light moves faster for this observer. We expect that students show they can interpret the consequences of relative velocity for the phenomenon of time dilation by mentioning that the effect of time dilation increases with increasing relative speed.

\section{Empirical Evaluation}

\subsection{Method}

In order to test whether our approach would be feasible in principle, and to gain insight in possible student responses, we conducted an empirical evaluation with small groups of students, similar to the teaching experiment described by Komorek and Duit (2004). In such a teaching experiment, the researcher has a dual role of teacher and clinical interviewer. When student responses are unclear, the teacher will probe students' reasoning by asking for further explanation. In this study, our focus was on the functioning of the educational design, rather than individual student learning trajectories. To explore the potential of the teaching approach under optimal conditions, we worked with students who volunteered to participate outside regular class time. In order to gain a more complete picture of how the design can function in teaching practice, the experiment has been performed with multiple groups of students, and with students varying in proficiency levels (cf., Bakker, 2018; Komorek \& Duit, 2004; Plomp \& Nieveen, 2013a; Plomp \& Nieveen, 2013b).

Participants In total, 30 students volunteered to participate in the research. All participants were $11^{\text {th }}$ grade students in two different schools at the pre-university level, and had opted for the science track. Different versions of the design were performed with 12 groups in total, with each group consisting of 2-4 students. Groups were composed based on availability of the students at the given time slots. The experiment consisted of 3-4 lessons per group; lessons took place once a week in a free hour of the students. In this paper, we present data of the learning process of five groups (15 students in total) who worked with the final version of the teaching and learning materials as described in Section 3. The physics proficiency of the participating students as reported by their regular physics teacher is presented in Table 2. For the purpose of reporting, all students were given an alias. All students signed a consent form for participating in the study, collecting their notebooks and recording the lessons on video.

Implementation of HLT In this study, the first author takes the role of the teacher, following the teacher actions as described in the HLT as closely as possible. Students and teacher sit in a circle around a table. On the table, there is a central ED. Students also have individual notebooks and pencils. The tasks are presented verbally by the teacher. The supporting EDs are available in two forms: an individual notebook for each student and a 
Table 2 A short characterization of each group

\begin{tabular}{ll}
\hline Group number & Proficiency level in physics \\
\hline $\begin{array}{l}\text { Group A } \\
\text { Group B }\end{array}$ & $\begin{array}{c}\text { Thomas and Martijn. Both belong to the most proficient students in their class } \\
\text { Lisa, Daniel, and Anne. Lisa is a proficient student; Daniel's and Anne's proficiency is } \\
\text { Group C }\end{array}$ \\
Lroup D & $\begin{array}{c}\text { Laura, Kevin, and Iris. All students have an average proficiency } \\
\text { average proficiency. Tessa has a low proficiency }\end{array}$ \\
Group E & $\begin{array}{c}\text { Kelly, Jeroen, and Bart. Bart is a highly proficient student, Kelly and Jeroen have a low } \\
\text { proficiency }\end{array}$ \\
\hline
\end{tabular}

bigger central version in which the students could collaborate. This central ED is placed on a portable whiteboard. Students can construct light propagation in this ED through placing small magnets in it. The intention of this central ED is both to stimulate collaboration and exchange of ideas between students and to allow students to have a dynamic interaction with the representation. The individual notebooks are used to write down/draw the outcome of the collective effort.

In an earlier version of the intervention, we only presented students with EDs in individual notebooks in which they could draw light propagation. There were two drawbacks to offering only individual EDs. First, students showed little interaction. Since a small groups approach had been chosen to stimulate student interaction, and to study student learning through their conversations, this was not a desirable effect. Second, students lost track of their reasoning process due to crossing out little mistakes in their drawing. Therefore, we offered an extra ED to work on as a group in the final version of the intervention. We observed that students were discussing their ideas with each other, while constructing light propagation in the ED. Offering the ED on a whiteboard, while students could construct light propagation by placing tiny magnets in the diagram was also forgiving on small errors. Instead of crossing out a wrong drawing, creating a messy situation, students could simply shift the magnet to the intended position and keep track.

Overall, participants were highly involved in the activities. Students had a good recollection of the previous lessons, considering a week with other lessons and homework had passed in the meantime. They could describe in detail what they did a week ago and what conclusions they had reached. Students were actively participating in the lessons and showed an interest in the materials. They were motivated to learn more about relativity and to participate in the study. Subsequently to the tasks described in the HLT, we spent some time with the students deriving the formulas for time dilation and length contraction. All students participated for the full 8 lessons of the HLT and these additional lessons in their free time, except for one student. This student had to quit after 5 lessons because of changes in his schedule. Since there was no reward for staying and no penalty for dropping out, this shows students were committed to the study. SRT is one of 12 subjects in the Dutch upper secondary curriculum. The curriculum does not prescribe a specific number of lessons for each subject. Typically, teachers take 3-4 weeks per subject, which corresponds to 6-12 lessons. Our design fits well within these boundaries.

Data Collection In order to gain insight in students' reasoning and learning processes, we need to analyze their utterances and actions. To that end, all lessons were recorded on 
video, and all student notebooks were collected. The camera was directed at the tabletop to register students working on the collective ED. Sound was recorded with a tabletop microphone connected to the video-camera. Video data were transcribed, and, for each group, the video and notebook data were combined into one comprehensive description of the lessons (data-triangulation, Denscombe, 2014).

Data Analysis Subsequently, the descriptions were compared to the evidence of learning described in the HLT, to come to a description of actual student learning using a constant comparative method (Bakker, 2018; Cobb \& Whitenack, 1996; van der Wal et al., 2019). In this process, the HLT functioned as a theoretical guide to interpret student learning. With the HLT as a guide for comparing hypothetical and actual student learning, we first identified examples and counterexamples of the expected learning described in the HLT. In those instances of counterexamples of expected learning, we tried to identify clues in student interaction with the tasks thus far to explain why these student views would make sense from the student perspective. This analysis resulted in a detailed account of student learning at the group level, describing and interpreting if and how the hypotheses on student learning as described in the HLT were met. Each step in the process of describing and interpreting student learning was checked by a second analyst. This analyst studied the raw data material, and checked if the extended description was complete, and if subsequent interpretations were consistent with the data. Disagreements were resolved through discussion (cf., Akkerman et al., 2008).

Here follows a short illustration of how the analysis was performed. The extended description of the lessons was interpreted by the first author; these interpretations and conjectures on student learning were checked by the second analyst. This episode shows Lisa performing the first task of the HLT. Our hypothesis on student learning for this task, as formulated in the HLT, is that students solve the task using a light propagation model with a constant speed relative to the lamp or to the graph paper. The analysis therefore focusses on students' actions and utterances that support one of these models (or neither).

The video shows that Lisa places the magnet symbolizing the light flash two squares two the left of the sensor on the picture in the ED at $t=-1$ (see Fig. 6a). Therefore, the first author conjectured that Lisa reasons with a constant speed of light relative to the graph paper. Lisa then says: "You would say that it has to be like this, but I think that is strange" and continues: "He [the observer] stands here [picture of the ED at $t=0$ ] at the fourth square [distance measured from the right lamp], so then he [the magnet/light flash] is here [picture of the ED at $t=-1$ ] also at the fourth [square measured from the left lamp], so then I would move him [the magnet/light flash] two squares backwards [in the direction of the right lamp] because he [light] moves with two squares per time unit." (see Fig. 6b). This last quote and Lisa's actions led the first author to conjecture Lisa reasons with a constant speed of light relative to the lamp. This results in two contradicting conjectures about Lisa's pre-instructional light propagation model: a constant speed relative to the graph paper and a constant speed relative to the lamp. The first author then studied the rest of the episode to find out which conjecture is supported by her utterances.

In the teaching experiment, the teacher now took on the role of clinical interviewer and asked Lisa to proceed with the task for the left lamp. Lisa responded: "I had thought that here [picture $t=0$ of the ED] he [the light flash] has covered 8 squares. So, in the picture before $[t=-1$ in the ED] he has covered two [squares] less, light propagates with two squares per time unit, so I figured out that he [the magnet/light flash] would end up at six [squares distance from the left lamp]." Teacher: "And before that?" Lisa: "At the fourth [square from the left lamp]." The video shows that Lisa places magnets in the ED at two 
a

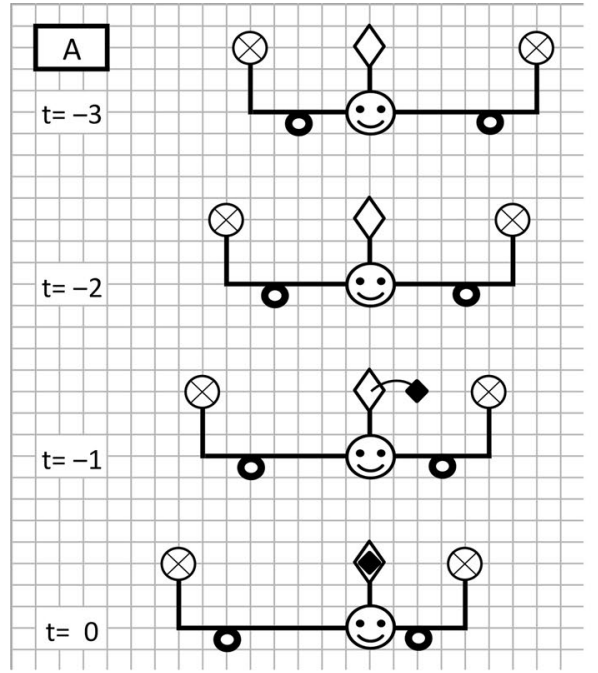

b

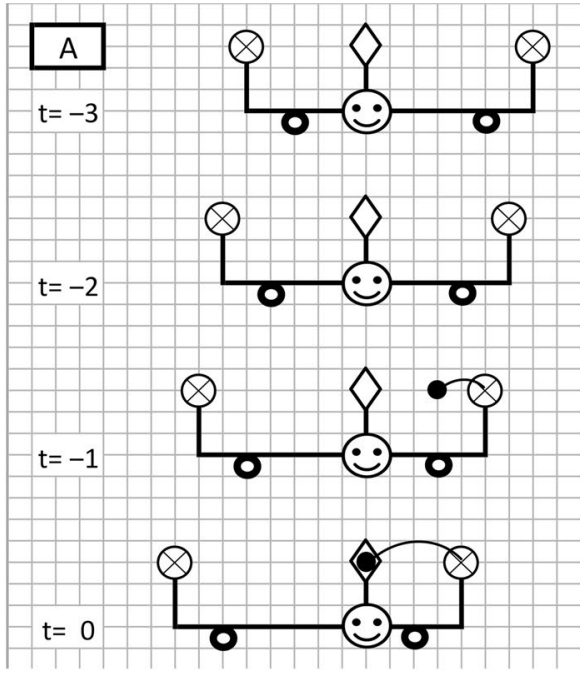

C

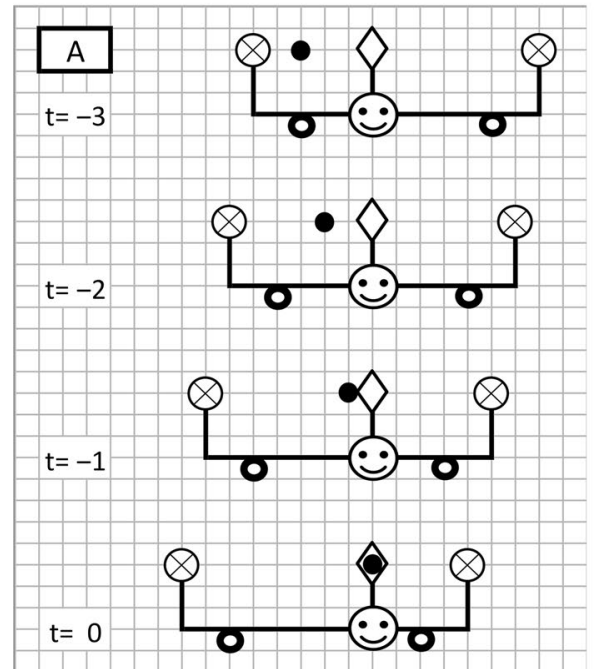

Fig. 6 Performance of Task 1 by Lisa. a Shows the initial positions of the magnet leading us to conjecture she reasoned with a constant speed relative to the graph paper. b Shows the positions of the magnets that led us to conjecture she reasoned with a constant speed relative to the lamp. c Shows the positions of the magnets that led us to dismiss our first conjecture and accept the second

squares from the left lamp in the picture of $t=-3$ and at the same position as the lamp in the picture of $t=-4$ (Fig. 6c). This reasoning is consistent with a propagation model constant relative to the lamp. Therefore, the conjecture that Lisa reasoned with a propagation model relative to the graph paper was dismissed and it was concluded that Lisa reasoned with a constant speed relative to the lamp. This interpretation of the lesson and Lisa's reasoning was presented to the second analyst, who checked the conjectures of the first author 
and agreed with her conclusions. However, throughout the analysis, both analysts remained alert to find examples and counterexamples to support or falsify this conclusion.

\subsection{Results}

In this section, we will present the empirical evaluation of the HLT. For each task, we will present what students do and say while performing the tasks, and our interpretation of this student behavior with respect to our learning aims. We will also reflect on the learning aims of the two parts of the HLT, and our overall learning aim.

\subsubsection{Part 1: Introducing the Need for a New Light Propagation Model-Becoming Aware of the Limited Predictive Value of Pre-instructional Light Propagation Models-Tasks 1 and 2}

The aim of these tasks is that students become aware of two things: One, (pre-instructional) light propagation models are a constant speed relative to something; and two, there are several options for this something (light source, graph paper). This should make it plausible for students to find out how light propagates.

All students engaged in the first two steps of hypothetical modelling with their initial propagation model: conform our expectations, they used this model to perform Task 1 and explained the propagation model to the teacher. Students referred to the reference frame of their pre-instructional model by showing how they constructed light propagation in the ED or they mentioned an element in the ED relative to which light had a constant speed:

Daniel (group B, reasoned with a constant speed relative to the graph paper): Light has a speed relative to the starting point.... final point, actually.

Lisa (group B, reasoned with a constant speed relative to the lamp): I would say relative to the point where it arrives, .... relative to the cart.

Daniel referred to the spot on the graph paper where the two light flashes end up at $t=0$ in the ED. For him, light has a constant speed relative to this point. Lisa counted the speed of light relative to the lamp. Some students also mentioned that the speed of light can be different from different points of view:

Martijn (group A, about a construction relative to the cart in ED A): Relative to the cart it [the speed of light] is two [squares per time unit], but for the human it is three or one, because of the relative movement.

When students dealt with two different propagation models (be it initial or formal), they often described the difference between the two as "taking the movement of the cart into account" or not (for example, see Lisa below). "Taking the movement of the cart into account" refers to light propagation with a constant speed relative to the lamp.

We expected that students might want to choose between two pre-instructional models if both were used to perform Task 1. Martijn is such a student, who considered both models and eventually preferred the paper model over the lamp model:

Martijn (Group A): This [constant speed relative to the graph paper] is more logical. Because it does not matter whether the cart is also moving once light is already moving, because the cart will never catch up with the light. [...] and if 
new light would be emitted that could get further than this light, then it [constant speed relative to the graph paper] would not be correct...but that is not the case.

Martijn referred to two principles that guide his choice. First, he reasoned that light emitted at one stage cannot be overtaken by light that has been emitted at a later point in time. Secondly, he thought the movement of the lamp should not influence the light flash after it has been emitted. The second principle guided him towards the paper model. Since this model was not contradictory to the first principle, he kept using this model.

As we expected, all students recognized the similarities between their pre-instructional model and one of the formal models and mentioned these similarities.

Daniel (group B): My approach is most similar to the wave [...] I only looked at the medium, not at the cart."

Lisa (group B): I think that my idea corresponds with the particles, because I take into account that the source moves [...] the lamp gives the velocity [to the light particles], or the medium.

In line with our expectations, all students but one could explain why both formal models are examples of a constant speed relative to something. They also used these models to perform Task 2 and concluded that the two models lead to different task outcomes. Only one student, Kevin, explicitly objected to one of the formal models because he did not recognize that the other model was an example of a constant speed as well. In addition, Kevin had trouble to correctly apply the formal model that did not correspond to his initial model. When fellow student Iris performed the task with the particle-like propagation model, he objected:

Kevin (Group C): Not convinced. I do not think that light moves that way. The speed of light is constant, also with particles, and now that is not the case.

Iris (Group C): [Relative] to the cart it is.

Kevin (Group C): Correct, but I would still look relative to the paper.

Kevin is very convinced of his initial model and therefore has difficulty to accept that speed can be constant relative to something else than the graph paper. Nevertheless, Kevin was absolutely aware of the difference between the two propagation models. We expected that the models leading to different task outcomes would create some tension between the models that made it plausible for students to consider different options for light propagation frames. The tension Kevin experienced was not intended in our design. However, it turned out to be a fruitful moment for Kevin to eventually accept the light postulate.

In the presentation of the wave-like formal model, light speed was defined relative to the "medium" which was represented by the squares of the graph paper in the ED. We expected this to be unproblematic for students. However, Daniel and Lisa were in doubt what element in the ED takes on this role. Instead of the graph paper, as intended, they chose the measuring device (sensor) to have the role of medium. Apparently, the students thought a medium is a kind of object, rather than something light (or sound) propagates through. Since the measuring device and observer are always at the same position in our task design, the students use both these terms.

Lisa applied her version of the wave-like model in ED B, where the measuring device moves relative to the graph paper but is stationary relative to the lamp. This led to confusion because the students expected that the two formal models would give different outcomes, similar to the results they obtained with their pre-instructional models in Task 1. 
Lisa constructed light propagation with a constant speed relative to the sensor, which led to the same results as a constant speed relative to the lamp:

Lisa (Group B): This is what we had figured out for the wave.

Daniel (Group B): But, isn't the other one [particle-like propagation model] the same?

This observation led to a discussion among the students how the wave-like propagation model should be applied. The students concluded the following:

Lisa (Group B): Each time, you have to take two steps relative to the light.... the observer.

Daniel (Group B): Yes.

The fruitful position Daniel and Lisa had for finding some tension between the models after preforming Task 1 crumbled because of the confusion they experienced with the wave-like formal model. Their focus shifted from interpreting the outcome of the task to correctly applying the models. As a result, the expected tension between different models predicting different outcomes did not arise for these students, and they erroneously concluded that the wave-like model defines light propagation relative to the observer.

Conform our design, all groups recognized that the two formal models led to different task outcomes. The group of Daniel and Lisa eventually reached this conclusion as well. We expected students would express the need to choose between the models. Except for Martijn, students did not express this need verbally. However, they agreed with the teacher that it is a plausible step to find out next which of the two models produces correct predictions. Therefore, after performing these two tasks, all students are aware that there is more than one option for the reference frame of light propagation models. As a consequence, students agree with the teacher that there is a need to take a further look into these models, but the majority of the students does not actively question the reference frame of the formal models or their pre-instructional model.

\subsubsection{Part 1: Introducing the Need for a New Light Propagation Model-Becoming Aware of the Limited Predictive Value of Pre-instructional Light Propagation Models-Task 3}

The aim of this task is that students reject both formal propagation models and accept they need to introduce a new propagation model.

All students performed the relative movement task correctly. In line with our expectations, the groups could link the experiments to the corresponding EDs as a result. Students experienced more difficulty with "translating" the outcome of the experiments to light propagation in the ED. Once they were successful with this, supported by the teacher, they could perform this part of the task independently for the second experiment. The following example illustrates how Jeroen summed up the reasoning process of his group:

Jeroen (Group E, about the MME in ED B): Those people [Michelson and Morley] are on earth. Let's imagine that the earth is that cart. They measure the same speed of light, so the two magnets [which the students use to construct light propagation in the ED] should go at the same speed relative to the earth.

Similar reasoning was observed in the other groups. Subsequently, all groups used the outcomes of the DSE and the MME to evaluate which formal model is confirmed by the 
experiments and which is rejected. This was also the case for Thomas and Martijn, who proposed an additional third propagation model, a constant speed relative to the observer:

Thomas (Group A, about the MME): For the researcher it [light] moves with two [squares] per time unit, so then he measures the same speed every time.

Teacher: Which models do remain now?

Martijn (Group A): Keep the observer and the particle.

For Kevin, interpreting the outcome of the MME turned out to be crucial in accepting that other propagation models than his pre-instructional particle like model are examples of a constant speed, something he struggled with in the previous task. Kevin suggested the particle-like model supports the outcome of the MME; however, he also experienced difficulty with accepting the light propagation mode confirmed by this experiment at the same time:

Kevin (Group C): This [the particle model in ED B] cannot be correct. The cart has a speed of one square each time. If you then look at the lightspeed, it is three and one.

Laura (Group C): But relative to the observer it is just two each time.

$[\ldots]$

Kevin (Group C): It is relativity theory, relative to what... Now [for the MME] we do not look at the graph paper.

The question "relative to what" in combination with information provided by experiments the experiments helped Kevin to accept the outcome of the experiment, and as a consequence, that other light propagation models are an example of a constant speed as well.

In line with our expectations, all groups obtained the overall task outcome: the MME confirms the particle-like model and rejects the wave-like model in situations like ED B, and the DSE confirms the wave-like model and rejects the particle-like model in situations like ED A. Therefore, all groups performed the third and fourth step of hypothetical modelling: evaluating the predictions and deciding to accept or reject a model.

We expected that the overall task outcome of the experiment would make it plausible for students to propose a new light propagation model. Two groups agreed with the teacher that a single new propagation model would be more practical than two models with a limited predictive value. In the three other groups, Groups A, C, and D, students showed some form of wondering how to proceed with this conclusion without a prompt from the teacher. For example, Thomas wondered if you can only make predictions with light propagation when you know which model works:

Thomas (Group A): Should you always find out first whether it [light] is a wave or a particle?

Iris's (group C) search for a single model that can be applied in all contexts, resulted in that she did not expect the outcome of the DSE. When Laura mentioned that this experiment confirmed the wave-like model, Iris objected:

Iris (Group C): I do not agree with that. It [light propagation] should be relative to the light source.

Teacher: Why do you think that it is relative to the light source?

Iris (Group C): Because we just excluded the wave [with the MME].

Iris was looking for a single explanation for light propagation: she expected that one of the two models was correct, and that both experiments would confirm this propagation 
model. She did not consider the option of needing a third model. Therefore, Iris disagreed when this expectation was not met. The teacher discussed with her that, even if they are not what you expect, experimental outcomes cannot be changed. After this discussion, she agreed that the DSE in fact supports the wave-like model:

Iris (Group C): Then Laura would be right, I think. [...] The particle-model is rejected now.

Eventually, all groups rejected the two formal models and students found it plausible to look for a new light propagation model.

\subsubsection{Part 2: Developing Confidence in the Light Postulate as a Propagation Model for Productive Reasoning-Task 4}

The goal of this task is that students formulate a new propagation model, the light postulate, and experience this model solves the problem of the limited predictive value of the formal models.

In line with the predicted learning in the HLT, one or more students in each group proposed their own version of the light postulate. The way students proposed the new model gave the impression this new model was obvious for them. They needed little time to formulate the new model and did not show a need to explain it. This shows students engaged in the first step of hypothetical modelling with the light postulate. Furthermore, our expectations that it is plausible for students they should find a new propagation model and that the previous task provided them with the necessary tools to propose the light postulate themselves are confirmed:

Bart (Group E): In both cases, it [the light flash] moves two squares relative to the observer.

Martijn (Group A): Relative to the observer, in that case it is always the same.

Iris (Group C): The waves in A and the particles in B? [...] in that case it is relative to the observer, two squares, the speed of light. [...] Then the speed of light is two squares each time, it does not matter in which situation.

Kevin (Group C): Yes, [...] the distance light covers in both situations is the same from the observer's point of view, and it moves the same distance from the observer.

Students expressed the new propagation model in different ways, but all of them mentioned the constant speed relative to the observer. Note that both Iris and Kevin, who had previously stuck to one of the formal models, now formulate the light postulate themselves.

As intended, the students either checked whether the new model works, or they had mentioned the observer already while interpreting the experimental outcomes in the ED. With these actions, students verified that the new model confirmed what they knew (the outcome of the experiments) and that it solved the problem of the limited predictive value of the two formal models. In group D, none of the students expressed tension or discomfort with the overall task outcome of Task 3. Nonetheless, one of the students proposed the light postulate:

Max (Group D): Could it be that light always moves with the same speed relative to you, the observer. [That] was the case here [points at task A] and also with the previous one, right?

Niels (Group D): This would be in accordance with A wave and B particle, but not with the rest. 
It seems Niels did not recognize that the new model did not need to agree with all the predictions of the previous models. This may explain why the students did not experience tension between the propagation models: they had not recognized that the two models exclude each other.

\subsubsection{Part 2: Developing Confidence in the Light Postulate as a Propagation Model for Productive Reasoning-Task 5}

The aim of this task was that students use the light postulate to make predictions in a new context and use it for a fruitful research program and discover its consequences for the time of an event.

Conform our expectations, all students but one used the light postulate to perform Task 5. The exception was Max. In his group, Max was the one who had proposed the light postulate during the previous lesson. Now, Max objected that the light postulate would be counterintuitive in the new situation of ED C. He proposed that a constant speed relative to the paper might suit better. The other students in the group corrected him and he agreed that this was not the model they decided upon last lesson. Max used the light postulate in ED D of Task 5.

All students obtained the overall task outcome that the observer of ED C and ED D assign different times to the same event, showing they could use the light postulate to start a fruitful research program. In line with our expectations, all students expressed this was counterintuitive.

Bart (Group E): Strange that they [the lamps] switch on at different times [for the two observers] while the light[speed] remains the same.

Nevertheless, 13 of the 15 students committed to the light postulate and showed the expected confidence in the new propagation model. This group includes Kevin, who had great difficulty to consider options other than his pre-instructional model at first. Students therefore engaged in the second step of hypothetical modelling with the light postulate. In each group, at least one student also explicitly engaged in the third step. Conform our expectations, these students described how the task outcome is a consequence of the light postulate and the relative speed between the two observers:

Jeroen (Group E): Because they [the two observers] move at different speeds. [...] Light moves relative to the observer, so if the one observer has a speed relative to the other, it [light propagation] will go in a different way.

Three students showed some doubt about the validity of the light postulate after performing this task. Two of them reconsidered their initial model as a more suitable alternative:

Martijn (Group A, about the task outcome): That is not logical, but... [...] he [the second observer] will find different values [for time] than the one who is moving. It's a little strange. [...] [If] the speed of light is the same for all observers, they disagree on the time the light was shot away. [In the next lesson] Or it is not correct that the speed of light is the same for all observers. Those experiments [DSE and MME] showed that it [constant speed relative to all observers] the case, so they [the observers] are both right. 
Daniel (Group B, about task outcome of ED C): This cannot be correct, because they both cover the same distance to arrive in the middle, so then they [the lamps] should also have turned on at the same time. Maybe we should look at the position of the light where the observer receives it.

Both students reflected on the counterintuitive task outcomes. Martijn said it was strange that both observers measured different times for the moment the lamp emitted the light flash. He realized that he should either accept this counterintuitive fact or reject the light postulate. The interpretation of the experiments of Task 3 helped him to accept the light postulate. Daniel struggled with the outcome of the first part of the task. He thought that, since the observer is in the middle between the two lamps, he should find that the light flashes were emitted simultaneously. Since this is not the outcome he obtained, he proposed his initial model again. Both these students performed step 4 of hypothetical modelling, although the decision of Daniel to reject the light postulate is not the learning outcome we intended. In conclusion, the tasks in this part resulted in the intended effect that across all participating groups one or more students proposed the light postulate and that all students in our sample used this propagation model to perform the tasks.

\subsubsection{Part 2: Developing Confidence in the Light Postulate as a Propagation Model for Productive Reasoning-Task 6}

The goal of this task is that students reason productively with the light postulate and explore the consequences of the light postulate for time intervals.

All students drew light propagation in ED E. Some students struggled with drawing light propagation in ED F: should the light go vertically upward (and miss the top mirror), or should it move in the horizontal direction as well and hit the top mirror in the middle? These students did not spontaneously use the idea that events that happen in one reference frame, also happen in another frame. Once this issue had been settled, some students thought that the speed of light is the same for both observers in the vertical direction, and the horizontal movement could be ignored. This was an unexpected interpretation of the task outcome. However, in line with our expectations, all students used the light postulate to interpret the graphs of the light path. This way, the students showed they are committed to the light postulate, the fourth step of hypothetical modelling. The students confirmed our predicted learning by concluding that light would need more time to move up and down for the observer studying the moving mirrors:

Kevin (Group B): The light goes different, so they [the observers] do not agree on the time.

Martijn (Group A): [The speed of light] is two squares per time unit, the distance is bigger than twelve [squares], so the time is bigger than six [time steps].

The intention of our design was that tension between the two formal models would fuel the need for students to start reasoning with the light postulate and accept this model over their pre-instructional models. We expected this tension to occur in the first part of our design. However, the group of Niels and Max experienced this tension in the second part and used it productively to choose the light postulate as the one and only propagation model. They considered two alternatives: light either moves faster or it will take more time:

Niels (Group D): Light goes faster. 
Max (Group D): Or it arrives later [...] It arrives at a later time, because we had agreed that the speed of light was the same.

Niels (Group D, in a subsequent lesson): For the other observer it is a zigzag pattern. In that case, observer A thinks that the light goes faster, or that it takes a longer time for the light to go up and down.

Teacher: Which of the two is it?

Niels (Group D): It takes a longer time, because light has a constant speed.

Max initially did not use the light postulate in Task 5. Here, in Task 6, he referred to the light postulate to convince Niels that light needs more time for the observer studying the moving light clock. In a subsequent lesson, Niels used this argument himself to choose the correct but counterintuitive answer. In conclusion, it seems that after performing the six tasks, all groups used the light postulate to interpret the light clock thought experiment and accepted the light postulate.

In addition, we expected that students would infer the consequences of a higher relative velocity between the two observers for time dilation. One group (group B) did not infer this by themselves. However, they agreed with the teacher when she talked the group through the reasoning process step by step. The other groups performed this reasoning by themselves. They did so by first describing or drawing the changes of the light path and then interpreting this new path for the duration of the process. Finally, they compared it with the outcome of a slower relative speed.

Teacher: What happens if the relative speed increases?

Thomas and Martijn both draw the light path with a smaller slope and as a consequence longer path: It [the time interval] becomes even longer.

\subsection{Contribution of Design Principles to Productive Reasoning with the Light Postulate}

Our design was guided by three principles: students should experience the need for a new light propagation model, that this new model leads to a fruitful research program, and that new knowledge can be developed through hypothetical modelling activities. In this section, we will briefly summarize how the TLS based on these principles contributed to students' learning, thus answering the second sub-research question. This summary is also presented in Table 3.

Our teaching and learning sequence consists of two parts, each covering three tasks. The overall aim of the first part is to make the need for a new light propagation model plausible for students. We found that all students engaged in hypothetical modelling with their preinstructional light propagation models, and that they experienced the limitations of their pre-instructional model. As expected, students realized the limitations of their models at different points in the task sequence, but in the end, all students agreed that a new light propagation model was needed. However, most students had to be prompted by the teacher to make this insight explicit. All in all, the activities of the first part resulted in the introduction of the light postulate becoming plausible for students.

The second part of our teaching and learning sequence aims for students to engage in productive reasoning with the light postulate. In Task 4, we found that students in all groups proposed a new propagation model, the light postulate, and in three groups actively compared this new propagation rule to their initial idea. In all groups, one or more students agreed that the light postulate solved the problems students experienced with their 
政

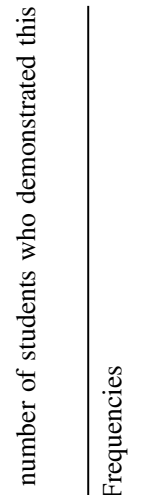

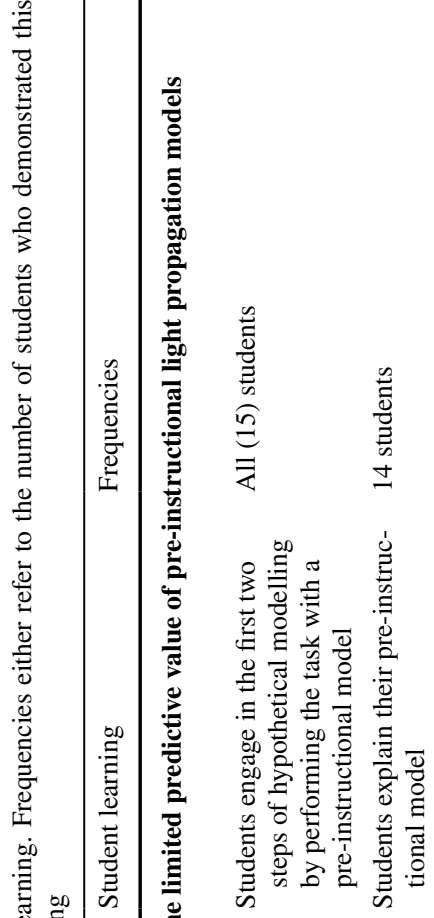

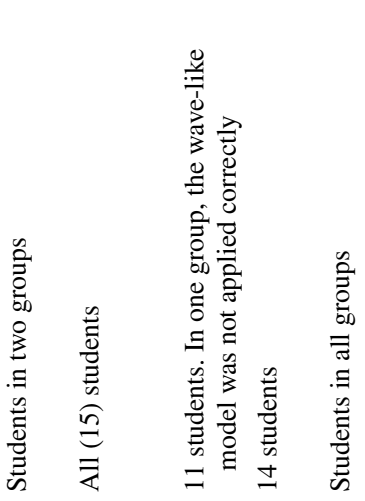

$\stackrel{\Xi}{\Xi}$

$\frac{\overrightarrow{0}}{2}$

馬

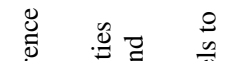

总

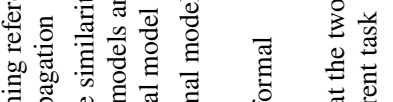

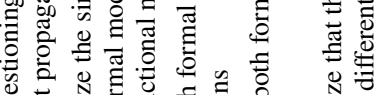

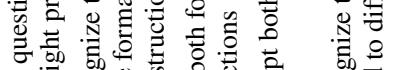

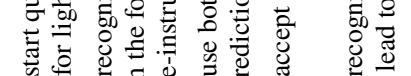

की

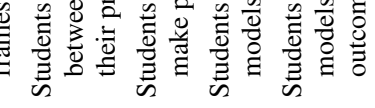

量

岁魚

范

可 :

ฐ क्ष

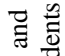

尊

ड़ँّ

के

on

军

范

氹

क्ष

०ै.

调灵

.

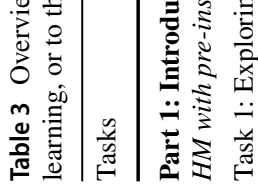

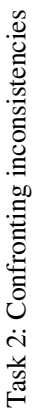

黛 Springer 


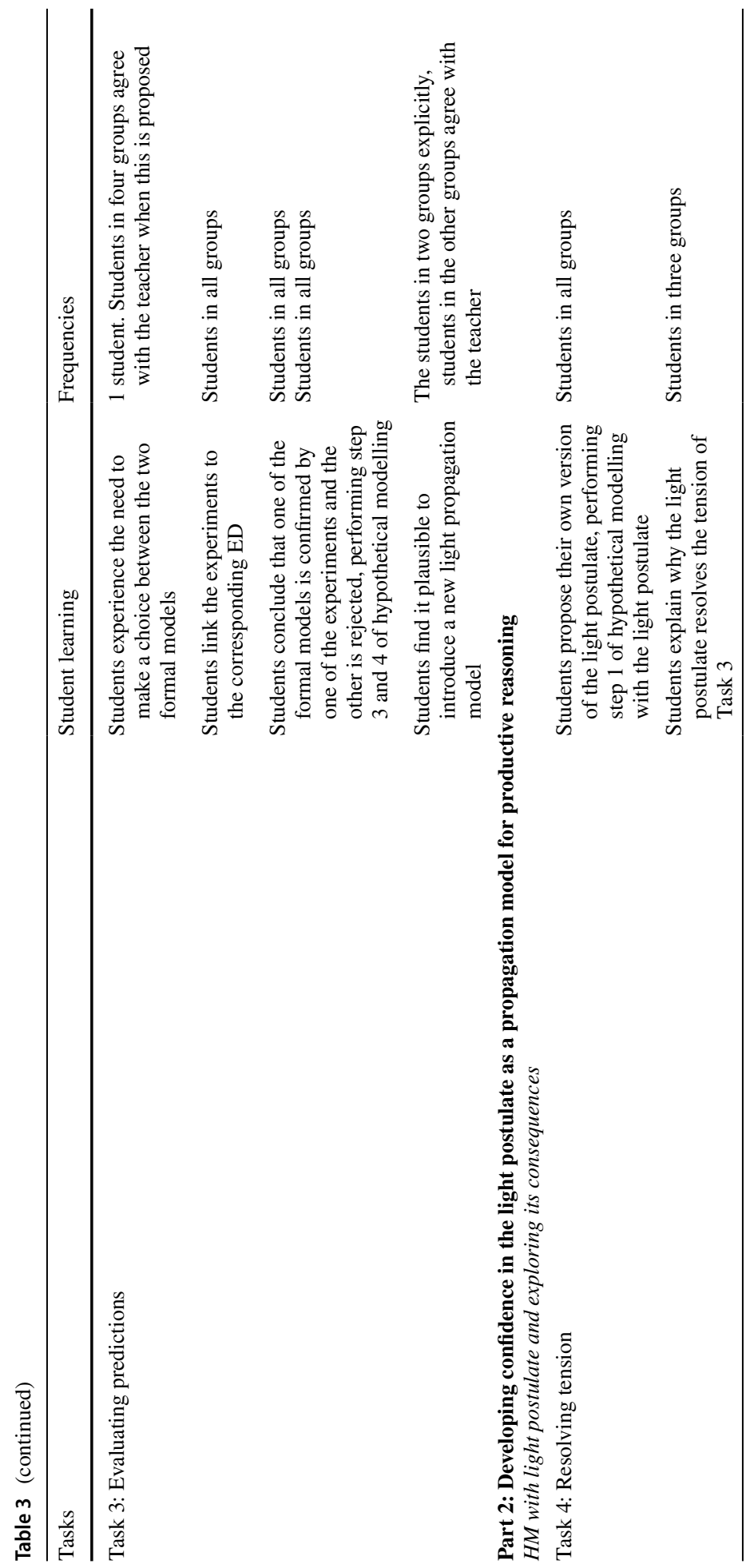




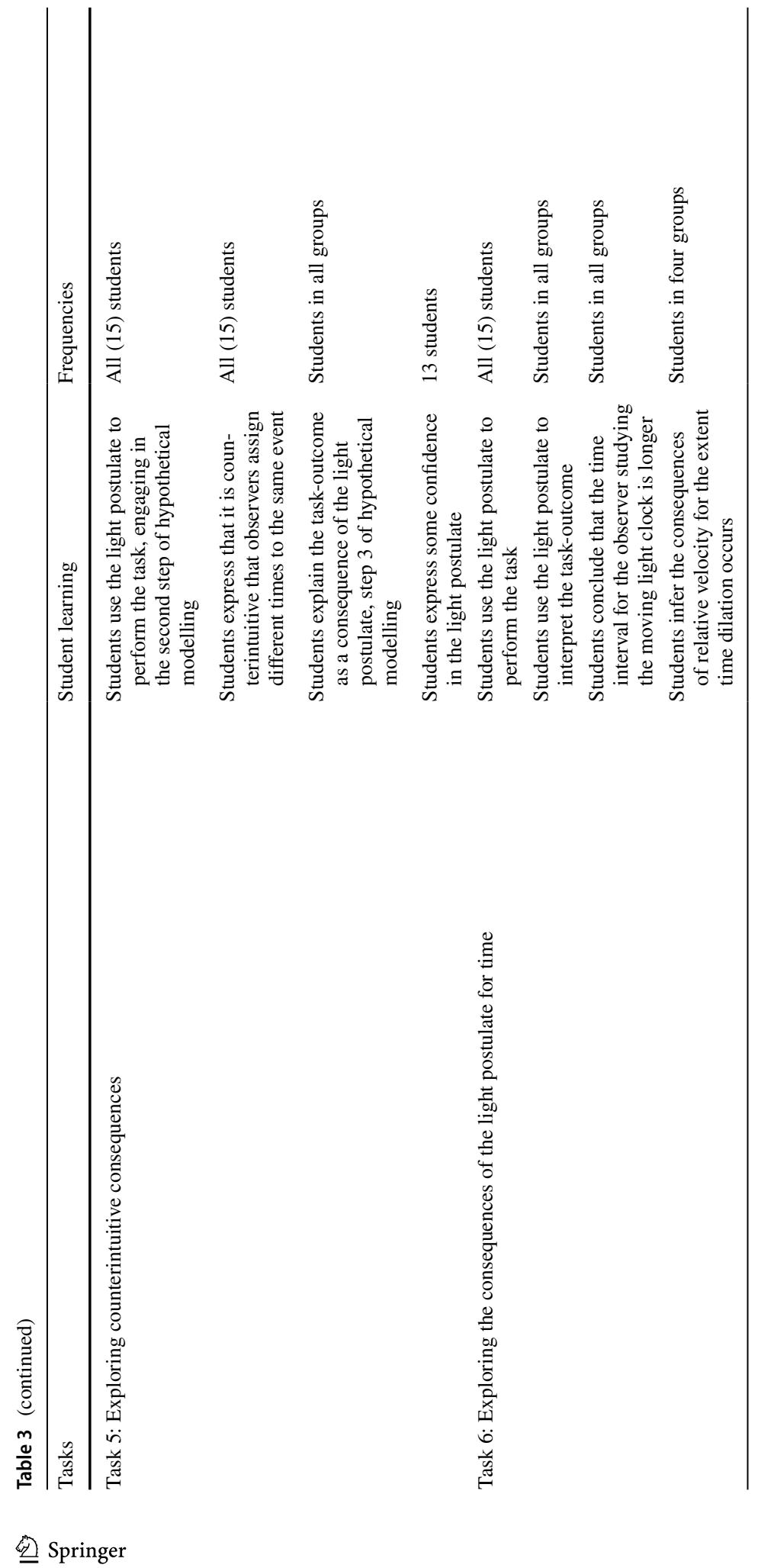


pre-instructional models. The results indicate that students who experienced a meaningful dissatisfaction with the initial propagation models were more likely to accept the light postulate. In Tasks 5 and 6, students derived counterintuitive relativistic phenomena while reasoning productively with the light postulate themselves. Student discourse indicates that during this activity, students came to accept the counterintuitive consequences of the light postulate. Because students could explain how the counterintuitive task outcome is a consequence of the initial settings of the thought experiment and the light postulate, they feel that the task outcome must be true, although counterintuitive. Almost all (13 out of 15 analyzed) students chose to accept the light postulate and continued using this postulate even if it led to counterintuitive outcomes. Furthermore, students used the light postulate to derive time dilation, engaging in productive reasoning with the light postulate. Thus, the activities in the second part of our design allowed students to experience that the light postulate solved the problem of the limited predictive value of their pre-instructional models and led to a fruitful research program. We conclude that, because students could explain how the counterintuitive task outcome is a consequence of the initial settings of the thought experiment and the light postulate, they feel that the task outcome must be true, although counterintuitive.

The development of SRT is operationalized in the design through activities that require students to engage in hypothetical modelling. In these activities, students explored the consequences of their pre-instructional light propagation model and experienced its limitations. This way, the tasks provided students with a content-based reason to propose the light postulate. We found that all students could engage in hypothetical modelling, showing that our task design is suitable to inspire this mode of reasoning in secondary students. Relativistic thought experiments require students to reason consistently with the light postulate. Event diagrams supported students in stepwise reasoning with light propagation in the context of the thought experiment. As a result, they were able to perform the deductive reasoning to obtain the outcome of the TE themselves. Furthermore, the visualization offered a shared object for students to express their reasoning and therefore supported discussing their ideas on light propagation, the reasoning process and the overall task outcome.

To sum up, our findings illustrate that the teaching and learning sequence indeed inspired all students to engage in hypothetical modelling and resulted for almost all students in productive reasoning with the light postulate.

\section{Conclusion and Discussion}

Drawing on the Model of Educational Reconstruction (Duit et al., 2012), we proposed a content structure for instruction of SRT. This content structure formed the basis for our design of a teaching and learning sequence that maintained the characteristics of the reasoning process of SRT to introduce relativistic concepts. This TLS was presented in the form of a hypothetical learning trajectory with hypotheses about the learning process to be seen. Finally, this HLT was evaluated in a teaching experiment with small groups of students. In Section 2.4 and 4.3, we have answered our two sub-research questions. Here, we return to our main research question:

How can learners in secondary education develop a conceptual understanding of SRT through engaging in a process of physics theory development? 
The students participating in our study developed a conceptual understanding of SRT. Thirteen out of 15 students developed confidence in the light postulate as a propagation model for productive reasoning. They used the light postulate to derive other relativistic concepts and, in this process, interpreted velocity, the time of an event and duration relative to a specific reference frame. In the lessons, students engaged in hypothetical modelling activities with their pre-instructional light propagation model and the light postulate. Einstein also drew on this style of scientific reasoning when he introduced SRT in his article Zur Elektrodynamik bewegter Körper. Furthermore, the process of theory development was reconstructed for students by carefully designing the tasks so students could experience a mismatch between two theoretical ideas. Introducing SRT through this process of theory development starting from a meaningful dissatisfaction with their pre-instructional ideas was a fruitful approach to introduce SRT to secondary students. Therefore, we have proposed a successful approach to achieve productive reasoning with the light postulate and let students experience that the concepts of SRT are interconnected. However, the learning process that led to these learning outcomes varied between students, as a short summary of the learning process of Martijn and Kevin illustrates.

Martijn proposed three different light propagation models while working with the initial task. This way, he created friction between theoretical ideas by himself. The tasks helped him to dismiss two of these initial models on content-based reasons and to choose the light postulate as final light propagation model. He then used the light postulate to derive the relativity of simultaneity and time dilation and reasoned with the consequences of higher relative velocities on the phenomenon of time dilation.

Kevin showed a strong preference for his pre-instructional light propagation model. $\mathrm{He}$ did not accept that a different propagation model was also an example of constant speed, just a constant speed relative to something else. Kevin therefore did not realize at the expected moment that there were more options for constant propagation. However, he did come to this realization in a different task. The outcome of the experiments helped him to realize that there were more possibilities for a reference frame of constant propagation than just his preferred frame. He later used the overall outcomes of the two experiments to formulate the light postulate himself. Although the consequences of the postulate were still counterintuitive to Kevin, he kept using this new propagation model in subsequent tasks and he used the light postulate to derive time dilation in the light clock thought experiment.

Bakker (2018) mentions that HLTs are sometimes seen as rigid and as forcing students and teachers to follow one strict learning path. Although this criticism is not shared by early implementers of HLTs (Bakker, 2018; Simon, 1995), we paid special attention to avoiding this unwanted feature of HLT in our design. Specifically, we designed the tasks in such a way that students could come to the realization of the limitations of their preinstructional model while working with different tasks, without stopping the flow of the lesson series. The learning paths of Martijn and Kevin illustrate that the design did allow for such differences in student learning. These learning paths also show that students did try to develop a consistent physics understanding of the world, and that carefully designed tasks can help these students to bridge the gap between their pre-instructional ideas and physics concepts.

One of our learning aims was to engage students in hypothetical modelling. Although our task design was successful in supporting students to attain this learning goal, we also acknowledge it is possible to improve our task design even further to support students in this endeavor. First, some students experienced difficulty with reflecting on the overall task outcome because they were unsure about their reasoning with one of the light propagation models. We therefore propose to make a clear distinction between these two reflective 
activities. Before reflecting on the overall task outcome, students and teacher need to check if they reasoned consistently with the light propagation model at hand.

Second, students engaged in hypothetical modelling activities, but we did not make this explicit to them. If we want students to become aware of nature of science aspects in science education, there should be explicit attention not only on what they have learned but also on how they (and scientists) reason (Lederman, 1992). Furthermore, this awareness may also contribute to overcome two difficulties some students faced while working with our tasks. Some students experienced difficulty to reason with one of the formal models. Underlining hypothetical aspect may help these students to overcome their initial hesitation: They are not required to be committed to the model, and may later reject it. The students are only asked to explore what the task outcome would be, were the model to be correct.

In the second task, all students recognized that the outcomes of both models are different. However, not all students realized that, as a consequence, the two models cannot be true at the same time. This issue may also benefit from underlining the hypothetical aspect of the models: both models are plausible, and we temporarily assume them to be true. Subsequently, we can support students with the interpretation of this hypothetical aspect: although both models can be true, they cannot both be true at the same time.

A well-known problem with learning relativity is that students do not distinguish between the occurrence and the observation of the event (Scherr et al., 2001). We did not encounter this learning problem with our students. Our task design may have contributed to avoiding this misconception. In the first tasks, students would be presented with the observation of an event and they had to figure out when the event occurred in a specific reference frame. In the final task, students worked with an intelligent observer who compensated for signal travel time. The tasks confronted students with the difference between the occurrence and the observation of an event. It would be interesting to explore if students keep making this distinction when it is not stressed by the task design.

We used thought experiments supported with event diagrams to bring the relativistic world to students. We used the thought experiment in a specific task design: students were free to choose the model with which they wanted to execute the thought experiment and students had to perform the deductive reasoning to obtain the outcome of the experiment themselves. However, one could argue that to truly perform a thought experiment, the student has to be free to pose the central question of the TE and to choose the basic settings of the TE as well. It may be clear that such an open application of TEs did not suit our learning aims. However, this means that we have given an example of how to use thought experiments to introduce relativistic concepts, and that it would be interesting to further explore how students could benefit from an open TE.

The design choice to visualize the TEs with event diagrams is a central feature in our teaching and learning sequence. It was important for our design that students would engage in the deductive reasoning with light propagation of the thought experiments themselves. The ED allowed students to do that by supporting step-wise reasoning with light propagation. For instance, computer simulations have been widely used to visualize relativistic contexts (Carr \& Bossomaier, 2011; De Hosson et al., 2010; Savage et al., 2007; Sherin et al., 2017). Because the reasoning with light propagation in these visualizations is often embedded in the algorithm, these types of visualizations do not support students in the deductive reasoning with light propagation. However, these visualizations allow students to tweak the initial settings of a thought experiment and explore the consequences of these basic settings on the outcome of the TE. We briefly explored this in Task 6 . Therefore, a combination of visualizations may be beneficial for students to learn SRT. 
We structured our design process with the Model of Educational Reconstruction. The model allowed and stimulated us to regard learning consequently in the coherence of theory, student learning difficulties, and learning aim. The model stresses the importance of reconstruction of the science content for education and as such provides certain liberties to not strictly follow the historical logic of theory development, but to draw on these ideas to make a didactical structure that makes sense from the perspective of a novice learner. Two tasks in our design reflect this aspect of MER. First, the use of the analogy between students' pre-instructional models and two historical models to inspire consistent reasoning whilst still keeping the connection with students' ideas. Second, the use of the outcome of the De Sitter Experiment and the Michelson-Morley Experiment (MME) to allow students to evaluate their predictions with these previously mentioned historical models. The MME has a different role in the history of physics, but we drew on its outcome in our didactical reconstruction to allow students to make sense of the outcome in a context they could understand.

It has been suggested that the concept of event plays a crucial role in student learning to "redefine space and time according to the new constraints of the theory that is the unsurpassable and constant speed of light" (Levrini, 2014). As we have shown in our theory analysis and what also follows from this quote, relativistic reasoning draws both on the concept of event and of absolute light propagation. For this introduction to SRT, we focussed on the light postulate, because it is a central theme in the Dutch curriculum goals for SRT. However, we do not wish to contradict the importance of the concept of event. Our intervention may even form a starting point to introduce the concept of event on content-based reasons from a student perspective, since students now have experienced that time and place do not have the same values for all inertial observers.

The Model of Educational Reconstruction furthermore underlines the importance of iterative design cycles to test and refine the educational design in the educational practice. To this end, Komorek and Duit (2004) suggest evaluating the design using a teaching experiment, working with small groups of students to find out learning patterns of students. In addition, we think it should always be an aim of designers and researchers of education to embed the design into real-life praxis. To make such larger scale evaluations useful, a sound basis of knowledge of possible learning paths of students is needed (Lijnse, 2001). With this research, we have provided the first, and crucial, step towards classroom evaluations. However, the current version of our design cannot be directly implemented in the classroom as it relies heavily on a teacher working closely with students individually to guide their reasoning. A subsequent design should address this issue to allow students to work more independently with the tasks, while keeping the feature that students can explore and challenge their ideas. In this version of the design, we have shown that students can follow different learning paths to obtain the learning goal; this should be retained in a new design. Finally, the students we worked with were highly motivated to participate in the study. One could imagine that working with a classroom of students who did not volunteer to study relativity will face the teacher with some motivational issues as well (Bøe et al., 2018). We propose that the minds-on approach of our tasks may address these motivational issues and engage students in their learning process.

In conclusion, we have shown that a teaching sequence based on hypothetical modelling with students' pre-instructional light propagation models engages students in the process of theory development of SRT and results in students gaining a conceptual understanding of SRT. This study provided a detailed account of possible student learning when working with the educational design, thus providing a proof of principle. We expect this approach can also be fruitful in the classroom when the earlier mentioned concerns are addressed 
and improvements implemented to make the design suitable for larger groups. We intend to implement these adaptations and see if these results can be reproduced in the classroom in our next study.

Acknowledgements The authors would like to thank Marianne Verhaart for her help with the analysis, and all the students who participated in this study for their time and effort.

Funding This work was supported by the Dutch Ministry of Education, Culture and Science under Grant OCW/PromoDoc/1065001.

\section{Declarations}

Conflict of Interest The authors declare that they have no conflict of interest.

Open Access This article is licensed under a Creative Commons Attribution 4.0 International License, which permits use, sharing, adaptation, distribution and reproduction in any medium or format, as long as you give appropriate credit to the original author(s) and the source, provide a link to the Creative Commons licence, and indicate if changes were made. The images or other third party material in this article are included in the article's Creative Commons licence, unless indicated otherwise in a credit line to the material. If material is not included in the article's Creative Commons licence and your intended use is not permitted by statutory regulation or exceeds the permitted use, you will need to obtain permission directly from the copyright holder. To view a copy of this licence, visit http://creativecommons.org/licenses/by/4.0/.

\section{References}

Abiko, S. (2005). The light-velocity postulate. Science \& Education, 14(3-5), 353-365.

Akkerman, S., Admiraal, W., Brekelmans, M., \& Oost, H. (2008). Auditing quality of research in social sciences. Quality \& Quantity, 42(2), 257-274.

Amin, T. G., \& Levrini, O. (2017). Converging perspectives on conceptual change: Mapping an emerging paradigm in the learning sciences. Routledge.

Aslanides, J. S., \& Savage, C. M. (2013). Relativity concept inventory: Development, analysis, and results. Physical Review Special Topics-Physics Education Research, 9(1), 010118.

Bakker, A. (2018). Design principles, conjecture mapping, and hypothetical learning trajectories. Design research in education (pp. 46-67). Routledge.

Bandyopadhyay, A. (2009). Students' ideas of the meaning of the relativity principle. European Journal of Physics, 30(6), 1239.

Bøe, M. V., Henriksen, E. K., \& Angell, C. (2018). Actual versus implied physics students: How students from traditional physics classrooms related to an innovative approach to quantum physics. Science Education, 102(4), 649-667.

Carr, D., \& Bossomaier, T. (2011). Relativity in a rock field: A study of physics learning with a computer game. Australasian Journal of Educational Technology, 27(6)

Cobb, P., \& Whitenack, J. W. (1996). A method for conducting longitudinal analyses of classroom videorecordings and transcripts. Educational Studies in Mathematics, 30(3), 213-228.

College voor Toetsen en Examens. (2018). Natuurkunde VWO - syllabus centraal examen 2020. Retrieved from https://www.examenblad.nl/examenstof/syllabus-2020-natuurkundevwo/2020/f=/natuurkunde_ 2_versie_vwo_2020.pdf

de Sitter, W. (1913). A proof of the constancy of the velocity of light. In KNAW, Proceedings, 15II, 1297-1298.

Denscombe, M. (2014). The good research guide: for small-scale social research projects. McGraw-Hill Education.

de Hosson, C., Kermen, I., \& Parizot, E. (2010). Exploring students' understanding of reference frames and time in Galilean and special relativity. European Journal of Physics, 31(6), 1527.

Dimitriadi, K., \& Halkia, K. (2012). Secondary students' understanding of basic ideas of special relativity. International Journal of Science Education, 34(16), 2565-2582. 
Disessa, A. A. (1996). What do "just plain folk" know about physics. The Handbook of Education and Human Development: New Models of Learning, Teaching, and Schooling, 709-730.

Driver, R., Asoko, H., Leach, J., Scott, P., \& Mortimer, E. (1994). Constructing scientific knowledge in the classroom. Educational Researcher, 23(7), 5-12.

Duit, R., Gropengiesser, H., Kattmann, U., Komorek, M., \& Parchmann, I. (2012). The model of educational reconstruction-A framework for improving teaching and learning science. Science education research and practice in Europe (pp. 13-37). Springer.

Einstein, A. (1905). Zur Elektrodynamik bewegter Körper. Annalen Der Physik, 322(10), 891-921.

Einstein, A. (1952a). On the influence of gravitation on the propagation of light. (W. Perret \& G. B. Jeffery, Trans.) In The principle of relativity: A collection of original papers on the special and general theory of relativity (pp. 111-164). Dover Publications, Inc. (Translated from Die Grundlage der allgemeinen Relativitätstheorie, Annalen der Physik, 49, 1916)

Einstein, A. (1952b). On the electrodynamics of moving bodies (W. Perret \& G. B. Jeffery, Trans.) In The principle of relativity: A collection of original papers on the special and general theory of relativity (pp. 37-65). Dover Publications, Inc. (Translated from Zur Elektrodynamik bewegter Körper, Annalen der Physik, 17, 1905)

Einstein, A. (1961). Relativity: The special and general theory: A popular exposition. Crown Publishers Inc.

Einstein, A. (1979). Autobiographical notes (Paul Arthur Schilpp, Trans.). Autobiographical notes. A centennial edition. Open Court Publishing Company.

Gim, J. (2016). Special theory of relativity in South Korean high school textbooks and new teaching guidelines. Science \& Education, 25(5-6), 575-610.

Gopnik, A., \& Wellman, H. M. (2012). Reconstructing constructivism: Causal models, Bayesian learning mechanisms, and the theory theory. Psychological Bulletin, 138(6), 1085.

Gousopoulos, D., Kapotis, E., \& Kalkanis, G. (2016). Students' difficulties in understanding the basic principles of relativity after standard instruction. In J. Lavonen, K. Juuti, J. Lampiselkä, A. Uitto, \& K. Hahl (Eds.), Electronic Proceedings of the ESERA 2015 Conference. Science education research: Engaging learners for a sustainable future, Part 1 (co-ed. O. Finlayson \& R. Pinto), (pp. 169-175). University of Helsinki.

Guisasola, J., Solbes, J., Barragues, J., Morentin, M., \& Moreno, A. (2009). Students' understanding of the special theory of relativity and design for a guided visit to a science museum. International Journal of Science Education, 31(15), 2085-2104.

Helm, H., Gilbert, J., \& Watts, D. M. (1985). Thought experiments and physics education. Physics Education, 20(5), 211-217.

Hewson, P. W. (1982). A case study of conceptual change in special relativity: The influence of prior knowledge in learning. European Journal of Science Education, 4(1), 61-78.

Huygens, C. (1952). On rays propagated in straight lines (S. P. Thompson, Trans.). In Treatise on light (pp. 1-22). The University of Chicago Press. (Translated from Traité de la lumière, 1690, Leyden).

Kamphorst, F., Savelsbergh, E., Vollebregt, M., \& van Joolingen, W. (2021). Event diagrams - Supporting student reasoning in special relativity through thought experiments. In D. Blair \& M. Kersting (Eds.), Teaching Einsteinian Physics in Schools - An essential guide for Teachers in Training and Practice. Routledge.

Kamphorst, F., Vollebregt, M. J., Savelsbergh, E. R., \& van Joolingen, W. R. (2019). Students' pre-instructional reasoning with the speed of light in relativistic situations. Physical Review Physics Education Research, 15(2), 020123

Kattmann, U., Duit, R., Gropengiesser, H., \& Komorek, M. (1996). Educational reconstruction-Bringing together issues of scientific clarification and students' conceptions. Paper presented at the Annual Meeting of the National Association of Research in Science Teaching (NARST), St. Louis, 1-20.

Kind, P., \& Osborne, J. (2017). Styles of scientific reasoning: A cultural rationale for science education? Science Education, 101(1), 8-31.

Klomp, H. A. (1997). De relativiteitstheorie in Nederland: breekijzer voor democratisering in het interbellum [The theory of relativity in the Netherlands]. Epsilon Uitgaven.

Komorek, M., \& Duit, R. (2004). The teaching experiment as a powerful method to develop and evaluate teaching and learning sequences in the domain of non-linear systems. International Journal of Science Education, 26(5), 619-633.

Lakatos, I. (1976). Falsification and the methodology of scientific research programmes. Can theories be refuted? (pp. 205-259). Springer.

Lederman, N. G. (1992). Students' and teachers' conceptions of the nature of science: A review of the research. Journal of Research in Science Teaching, 29(4), 331-359.

Levrini, O. (2008). How students learn from multiple contexts and definitions: Proper time as a coordination class. Physical Review Special Topics-Physics Education Research, 4(1), 010107. 
Levrini, O. (2014). The role of history and philosophy in research on teaching and learning of relativity. International handbook of research in history, philosophy and science teaching (pp. 157-181). Springer.

Lijnse, P. (2001). Didactics of science: The forgotten dimension in science education research? Designing Theory-Based Teaching-Learning Sequences for Science Education (p. 125). Utrecht, the Netherlands: CD- $\beta$ Press.

Lorentz, H. A. (1952a). Michelson's interference experiment (W. Perret \& G. B. Jeffery, Trans.). In The principle of relativity: A collection of original papers on the special and general theory of relativity (pp. 3-7). Dover Publications, Inc. (Translated from Versuch einer Theorie der elektrischen und optischen Erscheinungen in bewegten Körpern, Leiden, 1895, 89-92).

Lorentz, H. A. (1952b). Electromagnetic phenomena in a system moving with any velocity less than that of Light (W. Perret \& G. B. Jeffery, Trans.). In The principle of relativity: a collection of original papers on the special and general theory of relativity (pp. 9-34). New York, NY: Dover Publications, Inc. (Reprinted from the English version in Proceedings of the Academy of Sciences of Amsterdam, $6,1904)$.

Matthews, R. (1994). Thought experiments. New York, NY: Routledge.

National Research Council. (2012). A framework for K-12 science education: Practices, crosscutting concepts, and core ideas. National Academies Press.

Michelson, A. A., \& Morley, E. W. (1887). On the Relative Motion of the Earth and of the Luminiferous Ether. Sidereal Messenger, 6, 306-310.

Newton, I. (1952) Opticks. or a treatise of the reflections, refractions, inflections and colours of light. Dover Publications, Inc. (Reprinted from the fourth edition, London, 1730)

Organisation for Economic Co-operation and Development. (2013). PISA 2012 assessment and analytical framework: Mathematics, reading, science, problem solving and financial literacy. OECD Publishing.

Panse, S., Ramadas, J., \& Kumar, A. (1994). Alternative conceptions in Galilean relativity: Frames of reference. International Journal of Science Education, 16(1), 63-82.

Pietrocola, M., \& Zylbersztajn, A. (1999). The use of the principle of relativity in the interpretation of phenomena by undergraduate physics students. International Journal of Science Education, 21(3), 261-276.

Plomp, T., \& Nieveen, N. (2013a). Educational design research part A: An introduction. Enschede, the Netherlands: SLO.

Plomp, T., \& Nieveen, N. (2013). Educational Design Research Part B: Illustrative Cases. Netherlands Institute for Curriculum Development.

Posner, G. J., Strike, K. A., Hewson, P. W., \& Gertzog, W. A. (1982). Accommodation of a scientific conception: Toward a theory of conceptual change. Science Education, 66(2), 211-227.

Ramadas, J., Barve, S., \& Kumar, A. (1996). Alternative conceptions in Galilean relativity: Inertial and noninertial observers. International Journal of Science Education, 18(5), 615-629.

Reiner, M., \& Burko, L. M. (2003). On the limitations of thought experiments in physics and the consequences for physics education. Science \& Education, 12(4), 365-385.

Saltiel, E., \& Malgrange, J. L. (1980). 'Spontaneous' ways of reasoning in elementary kinematics. European Journal of Physics, 1(2), 73.

Savage, C. M., Searle, A., \& McCalman, L. (2007). Real Time Relativity: Exploratory learning of special relativity. American Journal of Physics, 75(9), 791-798.

Scherr, R. E., Shaffer, P. S., \& Vokos, S. (2001). Student understanding of time in special relativity: Simultaneity and reference frames. American Journal of Physics, 69(S1), S24-S35.

Scherr, R. E., Shaffer, P. S., \& Vokos, S. (2002). The challenge of changing deeply held student beliefs about the relativity of simultaneity. American Journal of Physics, 70(12), 1238-1248.

Sherin, Z., Tan, P., Fairweather, H., \& Kortemeyer, G. (2017). "Einstein's Playground": An interactive planetarium show on special relativity. The Physics Teacher, 55(9), 550-554.

Simon, M. A. (1995). Reconstructing mathematics pedagogy from a constructivist perspective. Journal for Research in Mathematics Education, 26(2), 114-145.

van der Wal, N. J., Bakker, A., \& Drijvers, P. (2019). Teaching strategies to foster techno-mathematical literacies in an innovative mathematics course for future engineers. ZDM Mathematics Education, 51(6), 885-897.

van Oers, H., \& Wardekker, W. L. (1997). De cultuurhistorische school in de pedagogiek 171-214. Pedagogiek in Meervoud.

Velentzas, A., \& Halkia, K. (2013). The use of thought experiments in teaching physics to upper secondarylevel students: Two examples from the theory of relativity. International Journal of Science Education, 35(18), 3026-3049. 
Villani, A., \& Pacca, J. L. A. (1987). Students' spontaneous ideas about the speed of light. International Journal of Science Education, 9(1), 55-66.

Villani, A., \& Arruda, S. M. (1998). Special theory of relativity, conceptual change and history of science. Science \& Education, 7(1), 85-100.

Vosniadou, S. (1994). Capturing and modelling the process of conceptual change. Learning and Instruction, 4(1), 45-69.

Yildiz, A. (2012). Prospective teachers' comprehension levels of special relativity theory and the effect of writing for learning on achievement. Australian Journal of Teacher Education, 37(12), n12.

Publisher's Note Springer Nature remains neutral with regard to jurisdictional claims in published maps and institutional affiliations. 